\title{
Both the Establishment and Maintenance of Neuronal Polarity Require the Activity of Protein Kinase D in the Golgi Apparatus
}

\author{
Dong-Min Yin, Yan-Hua Huang, Yan-Bing Zhu, and Yun Wang \\ Neuroscience Research Institute and Department of Neurobiology, Key Laboratory for Neuroscience of Ministry of Education and Health, Peking \\ University, Beijing 100083, People's Republic of China
}

\begin{abstract}
Neuronal polarization requires coordinated regulation of membrane trafficking and cytoskeletal dynamics. Several signaling proteins are involved in neuronal polarization via modulation of cytoskeletal dynamics in neurites. However, very little is known about signaling proteins in the neuronal soma, which regulate polarized membrane trafficking and neuronal polarization. Protein kinase D (PKD) constitutes a family of serine/threonine-specific protein kinases and is important in regulating Golgi dynamics and membrane trafficking. Here, we show that two members of the PKD family, PKD1 and PKD2, are essential for the establishment and maintenance of neuronal polarity. Loss of function of PKD with inhibitor, dominant negative, and short interfering RNA disrupts polarized membrane trafficking and induces multiple axon formation. Gain of function of PKD can rescue the disruption of polarized membrane trafficking and neuronal polarity caused by cytochalasin D, which results in F-actin depolymerization. PKD1 and PKD2 are also found to be involved in the maintenance of neuronal polarity, as evidenced by the conversion of preexisting dendrites into axons on PKD inhibition. Unlike other polarity proteins, PKD does not interact with the cytoskeleton in neurites. Instead, PKD regulates neuronal polarity through its activity in the Golgi apparatus. These data reveal a novel mechanism regulating neuronal polarity in the Golgi apparatus.
\end{abstract}

Key words: neuronal polarity; protein kinase D; Golgi apparatus; membrane trafficking; axon formation; neuronal morphology

\section{Introduction}

Nearly every aspect of neuronal signaling depends on the development and maintenance of neuronal polarity (Craig and Banker, 1994), which has been studied most extensively using cultured embryonic hippocampal neurons (Dotti et al., 1988). After initially forming a lamellipodium (stage 1), neurons extend several short neurites that appear identical to one another (stage 2 ). The first evidence of polarity occurs when one neurite undergoes an extended period of growth, becoming the axon (stage 3 ).

The morphological polarization during the transition from stage 2 to stage 3 is definitively associated with reorganization of the trans-Golgi network (TGN) to plasma membrane trafficking (Bradke and Dotti, 1997; Tang, 2001). The mechanisms underlying the reorganization of membrane trafficking are complex and involve the destabilization of F-actin and the stabilization of microtubules in the axon ( $\mathrm{Yu}$ and Baas, 1994; Bradke and Dotti, 1999; Witte et al., 2008). Several molecules linked to regulation of the cytoskeleton have been implicated in different steps of neuronal polarization (Arimura and Kaibuchi, 2005, 2007; Wiggin et

Received March 26, 2008; revised July 9, 2008; accepted July 25, 2008.

This work was supported by grants from the National Natural Science Foundation of China, a grant from the Beijing Natural Science Foundation, and a grant from the National Basic Research Program (2007CB512100) of China.

Correspondence should be addressed to Prof. Yun Wang, Neuroscience Research Institute, Peking University, 38 Xueyuan Road, Beijing 100083, People's Republic of China. E-mail: wangy66@bjmu.edu.cn.

DOI:10.1523/JNEUROSCI.1291-08.2008

Copyright $\odot 2008$ Society for Neuroscience $\quad 0270-6474 / 08 / 288832-12 \$ 15.00 / 0$ al., 2005). At the same time, the Golgi apparatus also plays a pivotal role in neuronal polarization (Jareb and Banker, 1997; Zmuda and Rivas, 1998). Moreover, a recent study suggested that centrosome and Golgi localization in the early postmitotic stage guided where the axon would be formed (de Anda et al., 2005). Although the critical role of the Golgi apparatus in neuronal polarization is well established, very little is known about the active signals in the Golgi apparatus responsible for regulating membrane trafficking reorganization during neuronal polarization.

We address these issues with respect to the protein kinase D (PKD) family, which is composed of three members: PKD1/ $\mathrm{PKC} \mu, \mathrm{PKD} 2$, and $\mathrm{PKD} 3 / \mathrm{PKC} \nu$ (Rozengurt et al., 2005). We focused on this family for several reasons. First, it is required for TGN to plasma membrane transport in nonpolarized HeLa cells (Liljedahl et al., 2001). Second, PKD plays different roles in cell migration in different cell types (Prigozhina and WatermanStorer, 2004; Eiseler et al., 2007), and young neurons develop initial polarity by mechanisms analogous to those used by migrating cells (Bradke and Dotti, 1998). Third, PKD1 and PKD2, but not PKD3, specifically regulate basolateral membrane trafficking in polarized epithelial cells and might be important in the generation of epithelial polarity (Yeaman et al., 2004). Recent studies indicate that mechanisms governing polarity are conserved across cell types and suggest that some determinants of epithelial polarity may also play a role in neuronal polarization (Shi et al., 2003; Kishi et al., 2005; Shelly et al., 2007; Barnes et al., 
2007). Fourth, the protein domain and key amino acids of PKD1 involved in its Golgi localization are well elucidated (Maeda et al., 2001; Baron and Malhotra, 2002; Hausser et al., 2002).

Here, we demonstrate that PKD1 and PKD2, but not PKD3, are essential for the establishment and maintenance of neuronal polarity. These effects of PKD on neuronal polarity depend on its activity in the Golgi apparatus. Thus, we reveal a novel mechanism regulating neuronal polarity in the Golgi apparatus.

\section{Materials and Methods}

Antibodies and chemicals. We used the following antibodies and chemicals: rabbit polyclonal anti-PKD1 (sc-639; Santa Cruz Biotechnology), rabbit polyclonal anti-PKD2 (Calbiochem), mouse monoclonal antiGM130 (BD Biosciences Transduction Laboratories), monoclonal anticyclin-dependent kinase 5 (CDK5) antibody (Millipore; 05-364), mouse monoclonal anti- $\beta$ III tubulin (G712A; Promega), rabbit polyclonal antiMAP2 (Millipore Bioscience Research Reagents), mouse monoclonal anti-Tau-1 (Millipore Bioscience Research Reagents), mouse monoclonal anti- $\alpha$-tubulin (Sigma-Aldrich), mouse monoclonal anti- $\beta$-tubulin (Santa Cruz Biotechnology), mouse monoclonal anti-green fluorescent protein (GFP) (Santa Cruz Biotechnology). Tetramethylrhodamine isothiocyanate (TRITC), FITC-labeled goat anti-mouse IgG, FITC-labeled goat anti-rabbit IgG (Zhongshan Golden Bridge Biotechnology), and Alexa Fluor 350 Go-anti-rabbit IgG (Invitrogen) were used as secondary antibodies. The PKC inhibitors 12-(2-cyanoethyl)-6,7,12,13-tetrahydro13-methyl-5-oxo-5H-indolo[2,3-a]pyrrolo[3,4-c]carbazole (Gö6976) and bisindolylmaleimide I (BIM) were from Calbiochem. NGF and cytochalasin D were from Sigma-Aldrich. Post-Golgi membrane trafficking was detected with TRITC-labeled $\mathrm{C}_{5}$-ceramide Bodipy complexed to BSA (Invitrogen). Filamentous actin was detected with TRITC-labeled phalloidin (Sigma-Aldrich).

DNA constructs. GFP-PKD1 was constructed as described previously (Wang et al., 2004). All mutants of PKD1 were created using QuikChange kit (Stratagene), following instructions provided by the manufacturer. The different mutations were verified by DNA sequencing. The cDNA for PKD2 was amplified from adult rat brain and cloned into p-EGFP-C2 (Clontech). For dominant-negative (DN) PKD2, lysine 580 (codon AAG) was mutated to asparagine (AAC). GFP-wt-PKD3 and GFPK605N-PKD3 (dominant-negative PKD3) were kindly provided by Dr. Qiming Jane Wang (University of Pittsburgh, Pittsburgh, PA). The cDNA for C1a domain was amplified from the GFP-wt-PKD1 and cloned into p-EGFP-N1 (Clontech).

Neuronal culture and transfection. Hippocampal explants isolated from embryonic day 18 (E18) rat embryos (E16 mouse embryos were used in RNA interference experiments) were digested with $0.125 \%$ trypsin for 30 min at $37^{\circ} \mathrm{C}$, followed by trituration with pipette in plating medium (DMEM with 10\% fetal bovine serum). Dissociated neurons were plated onto $35 \mathrm{~mm}$ dishes coated with poly-D-lysine (Sigma-Aldrich) at a density of $1 \times 10^{5}$ cells per dish for adding inhibitors. After culturing for $2 \mathrm{~h}$, media were changed to Neurobasal medium supplemented with $2 \%$ B27 and $0.5 \mathrm{~mm}$ L-glutamate (Invitrogen), and the inhibitors were added. Transfection of neurons was performed before plating for $1 \mathrm{~h}$ at $37^{\circ} \mathrm{C}$ using Lipofectamine 2000 (Invitrogen) following instructions provided by the manufacturer. The neurons were centrifuged to remove the suspension and were resuspended with DMEM plus 10\% FBS. After plating the neurons for 3-4 h in dishes, media were changed to Neurobasal medium supplemented with 2\% B27 and $0.5 \mathrm{~mm} \mathrm{~L}$-glutamate. After $60 \mathrm{~h}$ in culture, neurons were fixed with $4 \%$ paraformaldehyde for $20 \mathrm{~min}$ at room temperature and processed for immunofluorescence. Cytochalasin $\mathrm{D}$ was added to the culture at $1 \mathrm{~d}$ in vitro ( $24 \mathrm{~h}$ after transfection). To do maintenance experiments, the neurons were plated at high density $\left(\sim 8 \times 10^{5}\right.$ cells per $35 \mathrm{~mm}$ dish $)$ and the transfection was done $2 \mathrm{~d}$ after plating.

RNA interference. The sequence of PKD1 short interfering RNA (siRNA) was as follows: 5' -CAGGAAGAGAUGUAGCUAU-3', which is conserved between human and mouse. The sequence of PKD2 siRNA was as follows: 5'-CAGUCAGAUCCAAGAGAAU-3', which is conserved between mouse and rat. Control siRNA was 5'-UUCUCCGAA-
CGUGUCACGU-3', which should not knockdown any known proteins. To do rescue experiments, the third base of each codon in the target sequence was mutated without changing the identity of the amino acids.

Immunofluorescence. Cells on culture dishes were washed with PBS three times, fixed with $4 \%$ paraformaldehyde for $20 \mathrm{~min}$, washed with PBS three times for $5 \mathrm{~min}$, permeabilized with $0.3 \%$ Triton X-100 for 30 $\mathrm{min}$, and washed with PBS three times for $5 \mathrm{~min}$. After blocking nonspecific binding with $10 \%$ goat serum in PBS for $1 \mathrm{~h}$ at room temperature, the cells were incubated with primary antibody overnight at $4^{\circ} \mathrm{C}$, washed with PBS three times for $5 \mathrm{~min}$, incubated with second antibody for $1 \mathrm{~h}$, and washed with PBS three times for $5 \mathrm{~min}$. Finally, the cells were mounted on slides, and the stained sections were examined with a Leica fluorescent microscope or a Leica TCS 4D confocal microscope using omnichrome air-cooled helium/neon lasers tuned to produce beams of 488 and $568 \mathrm{~nm}$. Where indicated, the neurons were incubated in microtubule stabilizing buffer (MSB) ( $2 \mathrm{~mm} \mathrm{MgCl}_{2}, 10 \mathrm{~mm}$ EGTA, $60 \mathrm{~mm}$ PIPES, pH 7.4), as a control, or in extraction buffer (1\% Triton X-100 diluted in MSB) for $10 \mathrm{~min}$ on ice before paraformaldehyde fixation.

Triton X-100 extraction and Western blot analysis. Two day in vitro (DIV2) neurons grown on dishes were washed twice with $\mathrm{Ca}^{2+}$ - and $\mathrm{Mg}^{2+}$-containing PBS and then incubated in extraction buffer $(150 \mathrm{~mm}$ $\mathrm{NaCl}, 10 \mathrm{~mm}$ Tris-HCl, pH 7.4, 1 mm $\mathrm{MgCl}_{2}, 0.5 \%$ Triton X-100, $0.1 \mathrm{~mm}$ PMSF, and a mixture of protease inhibitors) for $20 \mathrm{~min}$. The Triton $\mathrm{X}-100$-soluble and -insoluble fractions were denatured and subjected to SDS-PAGE, using 10\% running gels, and transferred to nitrocellulose membranes. After blocking with 5\% milk powder for $1 \mathrm{~h}$ at room temperature, the membranes were incubated with primary antibodies overnight at $4^{\circ} \mathrm{C}$. The membranes were then washed and incubated with HRP-conjugated secondary antibody (1:2000; goat anti-rabbit or -mouse; Bio-Rad Laboratories) and incubated for $1 \mathrm{~h}$ at room temperature. All washes were done using TBST ( $50 \mathrm{~mm}$ Tris- $\mathrm{HCl}, \mathrm{pH} 7.5,150 \mathrm{~mm}$ $\mathrm{NaCl}$, and $0.05 \%$ Tween 20 ), three times for $10 \mathrm{~min}$. Last, blots were detected using a chemiluminescence kit (Santa Cruz Biotechnology; sc-2048).

Coimmunoprecipitation. Cos7 cell lysates were immunoprecipitated with anti- $\beta$-tubulin antibody $(1: 100)$ at $4^{\circ} \mathrm{C}$ for $3 \mathrm{~h}$. DIV 2 neurons lysates were immunoprecipitated with anti-PKD1 (1:100) or anti-PKD2 (1:100) antibody at $4^{\circ} \mathrm{C}$ for $3 \mathrm{~h}$. Protein A-Sepharose CL-4B resin (GE Healthcare) was added to the samples, and the incubation was continued for an additional $12 \mathrm{~h}$, after which, samples were washed six times with TBS/ $0.1 \%$ Triton X-100. The final pellets were boiled with SDS-PAGE sample buffer and subjected to Western blot analysis.

Kinase assay. PKD1 autophosphorylation was determined in an in vitro kinase assay as described previously (Wang et al., 2004). A total of $150 \mu \mathrm{g}$ of protein from the lysate of young hippocampal neurons was used to do the in vitro kinase assay.

Labeling post-Golgi membrane trafficking. We used TRITC-labeled $\mathrm{C}_{5}$ ceramide Bodipy complexed to BSA to label the Golgi apparatus and post-Golgi membrane trafficking following the instructions of the manufacturer. Briefly, neurons washed once with DMEM free of phenol red (Invitrogen) were cultured with $5 \mu \mathrm{M} \mathrm{C} \mathrm{C}_{5}$-ceramide Bodipy at $4^{\circ} \mathrm{C}$ for 30 min, and then were washed twice with DMEM free of phenol red and cultured for an additional $30 \mathrm{~min}$ at $37^{\circ} \mathrm{C}$. Finally, the neurons were washed with PBS and examined by fluorescence microscopy.

Analysis of neuronal morphology. Dissociated neurons grown at low density were used to determine morphological characteristics. Neurons treated with inhibitors were randomly chosen, and transfected neurons were chosen based on GFP expression. The neurons were photographed with $40 \times$ magnification using a Leica phase contrast microscope (Leica). All processes and their branches were traced, their numbers were counted, and their lengths were measured using SPOT 32 software (Diagnostic Instruments). A cell was considered to have an axon if the length of one process was at least twice as long as any other process and was more than twice the diameter of the cell body or if this process was Tau-1-positive. Neurons were said to have normal polarity when they had only one axon. F-actin staining in the growth cones was analyzed with Image J.

Statistical analysis. All data were represented as mean \pm SEM. Comparisons between two groups were made using an unpaired $t$ test. Com- 

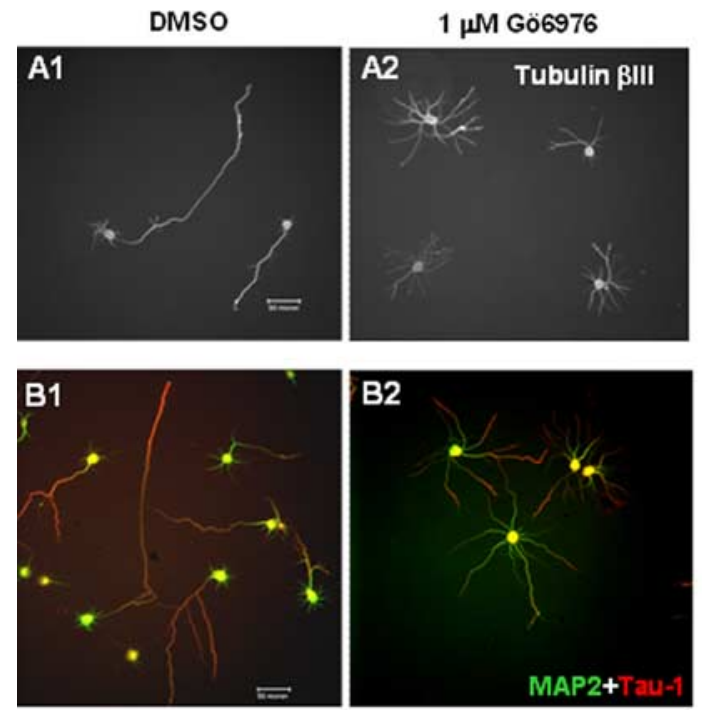
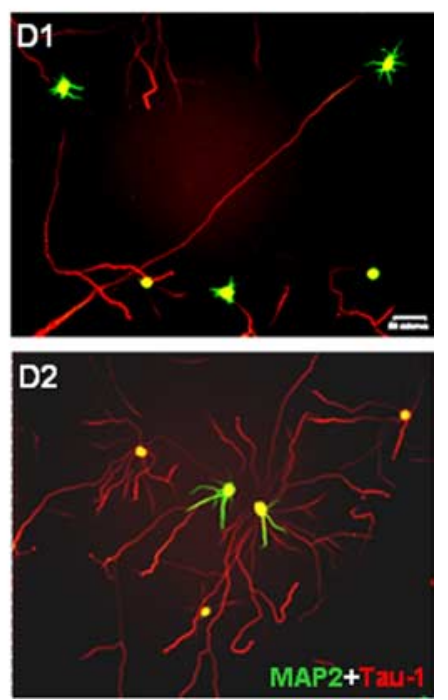

Figure 1. Effects of PKD inhibitor, Gö6976, on neuronal polarization. $A 1$, A2, Neurons treated with DMSO (A1) and $1 \mu \mathrm{M} \mathrm{Gö6976} \mathrm{(A2)} \mathrm{were} \mathrm{stained} \mathrm{with} \mathrm{anti-tubulin} \beta$ III. B1, B2, Neurons treated with DMSO (B1) and $1 \mu \mathrm{MG}$ Go976 (B2) were stained with anti-MAP2 and anti-Tau-1.C, Kinase assay of hippocampal neurons that were treated with DMS0 and $1 \mu \mathrm{M}$ Gö6976, and then were cultured in normal medium after washing out DMS0 and Gö6976. D1, D2, Neurons were treated with DMSO (D1) or $1 \mu \mathrm{m}$ Gö6976 (D2) for $2.5 \mathrm{~d}$, and then cultured for an additional $2.5 \mathrm{~d}$ in normal medium and stained with anti-MAP2 and anti-Tau-1. Scale bars, $50 \mu \mathrm{m}$.

parisons among more than three groups were made using one-way ANOVA analysis followed by the Newman-Keuls test. Data marked with three asterisks are significantly different from the control $(p<0.001)$. Data marked with two asterisks are significantly different from the control $(p<0.01)$.

\section{Results \\ Distribution of the Golgi apparatus and PKD1 in newly polarized DIV2 neurons}

To identify signaling proteins in the Golgi apparatus responsible for regulating polarized membrane trafficking, we first analyzed the radial distribution of the Golgi apparatus and the orientation of post-Golgi membrane trafficking in newly polarized hippocampal neurons. A line drawn between the center of mass of the neuronal soma and the emergence point of the axon was defined as $\theta=0^{\circ}$, and the somatic region was divided into four quadrants, as shown in supplemental Figure S1 $A$ (available at www.jneurosci.org as supplemental material). The probability of the Golgi being located in the quadrant of the neuronal soma immediately adjacent to the axon was $\sim 45 \%$ greater than for the other regions ( $n=110$ neurons; three experiments) (supplemental Fig. S1 B,D, available at www.jneurosci.org as supplemental material). Analysis with another Golgi marker, GM130, also indicated that, in general, the Golgi apparatus faced the axon in newly polarized neurons (supplemental Fig. S1E,F, available at www.jneurosci.org as supplemental material). Whenever the Golgi apparatus faced the axon (i.e., was in the first quadrant) or was adjacent to the axon (i.e., was in the second or fourth quadrants), post-Golgi membrane trafficking was directed toward the axon (supplemental Fig. S1B,C, arrows; available at www. jneurosci.org as supplemental material), consistent with the hypothesis that polarized post-Golgi membrane trafficking is an early determinant of neuronal morphological polarization (Bradke and Dotti, 1997).

Next, we analyzed the distribution of PKD1 in stage 1 to stage 3 hippocampal neurons using immunofluorescence. We found that PKD1 was distributed mainly in the cytoplasm, with high levels in the Golgi apparatus indicated by the colocalization of PKD1 and GM130 (supplemental Fig. S2A-C, available at www.jneurosci.org as supplemental material). We also found that basal activity of PKD1 was present in young hippocampal neurons but was abolished by $1 \mu \mathrm{M}$ Gö6976, an inhibitor of PKD (Gschwendt et al., 1996). A general PKC inhibitor, bisindolylmaleimide I (Martiny-Baron et al., 1993), had no such effect (supplemental Fig. S2D, available at www.jneurosci.org as supplemental material).

\section{PKD1 is required for neuronal polarization}

Hippocampal neurons were treated with the PKD inhibitor, Gö6976, or control vehicle, dimethyl sulfoxide (DMSO), and were cultured for $2.5 \mathrm{~d}$. Gö6976 decreased the number of neurons with a single axon in a dose-dependent manner and increased the number of neurons with multiple axons. Most of neurons treated with DMSO ( $80 \pm 1.18 \% ; n=292$ neurons; three experiments) had normal polarity, with a single axon and several dendrites indicated by the immunostaining with anti-tubulin $\beta$ III (Fig. 1A1) or anti-MAP2 (a dendritic marker) and anti-Tau-1 (an axonal marker) (Fig. 1 B1). In neurons treated with $1 \mu \mathrm{M}$ Gö6976, however, very few $(8.15 \pm 1.26 \% ; n=281$ neurons; three experiments) had normal polarity, whereas most had multiple axons (Fig. 1A2,B2). Neuronal polarity was quantitatively assessed by the ratio of the length of the longest neurite to the second, third, and fourth longest neurites. The ratio in neurons treated with 1 $\mu \mathrm{M}$ Gö6976 was significantly lower than in control neurons. In addition, the total number of neurites per neuron increased (DMSO, $3.83 \pm 0.28 ; 1 \mu \mathrm{M}$ Gö6976, $6.77 \pm 0.14$; three experiments), whereas the total neurite length per neuron was not affected by Gö6976 (DMSO, $449 \pm 84 \mu \mathrm{m} ; 1 \mu \mathrm{M}$ Gö6976, $537 \pm 58$ $\mu \mathrm{m}$; three experiments). This suggested that inhibition of PKD activity might specifically change membrane trafficking from a polarized mode to a nonpolarized mode, while not affecting the overall level of membrane trafficking.

To determine whether the effects of Gö6976 on neuronal polarization were reversible, Gö6976 was washed out at DIV2.5, and neurons were cultured for an additional $2.5 \mathrm{~d}$ in normal medium. As shown in Figure $1 C$, the kinase activity of PKD1 recovered after Gö6976 was washed out. However, the neuronal polarity did 

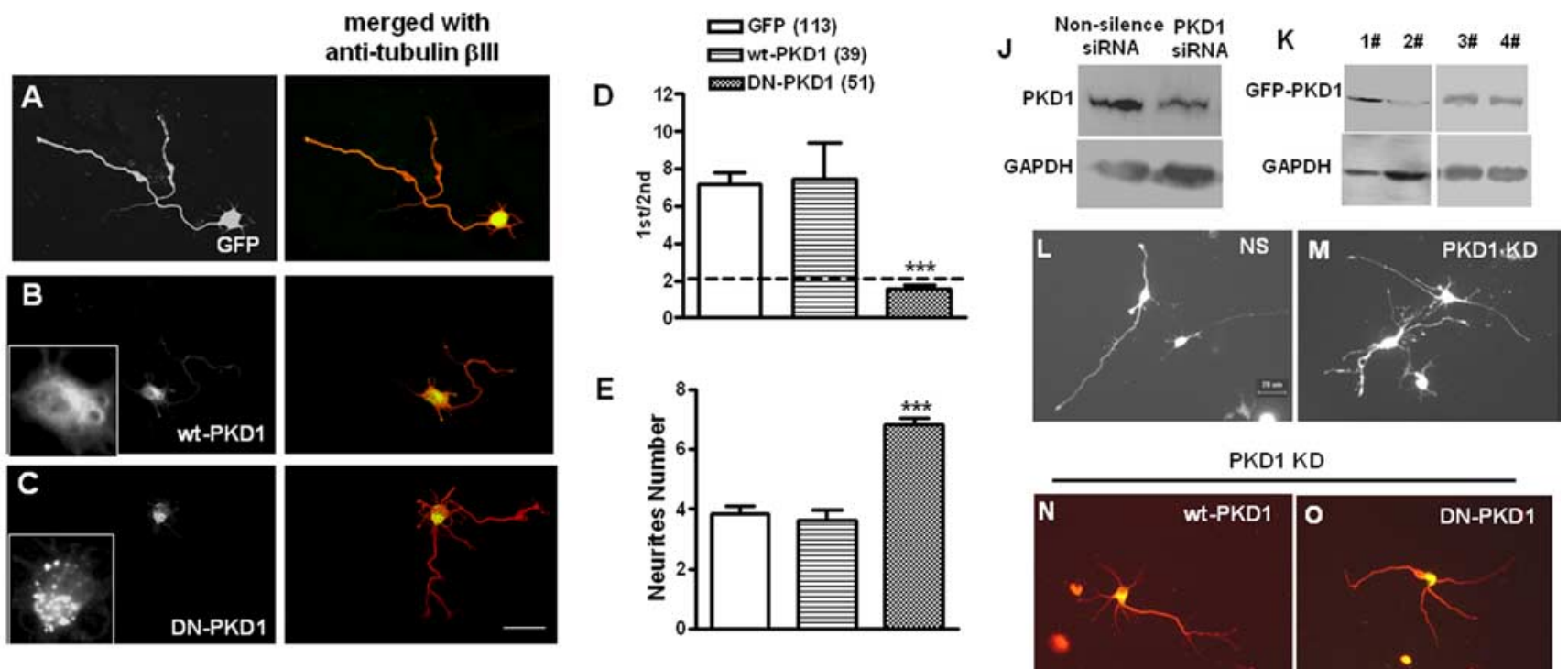

PKD1 KD
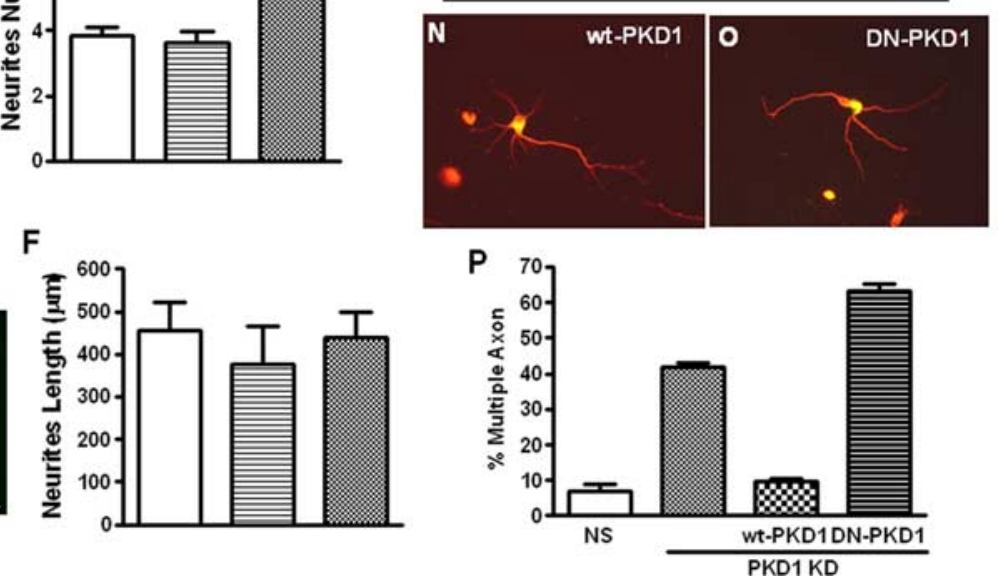

Figure 2. Effects of the dominant-negative PKD1 and PKD1 siRNA on neuronal polarization. $\boldsymbol{A}-\boldsymbol{C}$, Neurons were transfected with GFP (A), GFP-wt-PKD1 (B), and GFP-DN-PKD1 ( $)$, and were stained with anti-tubulin $\beta$ III. Enlargements show the different distribution of the three GFP fusion proteins in neurons. $\boldsymbol{D}-\boldsymbol{F}$, Ratio of the length of the longest neurite and the second long neurite $(\boldsymbol{D})$, total number of neurites per neuron $(\boldsymbol{E})$, and total length of neurites per neuron $(\boldsymbol{F})$ in neurons transfected with the indicated constructs. Bars indicate means \pm SEM of four independent experiments. Data were analyzed by ANOVA followed by Newman-Keuls multiple-comparisons test. ${ }^{* *} p<0.001$ compared with GFP group. Numbers in parentheses refer to the total neurons examined. G-I, Neurons transfected with GFP $(\boldsymbol{G})$, GFP-wt-PKD1 $(\boldsymbol{H})$, and GFP-DN-PKD1 (I) were stained with anti-Tau-1. J, PKD1 siRNA knock down the expression of endogenous PKD1 in mouse neuroblastoma N2a cells. $K$, Western blot analyses of GFP-PKD1 in HEK293 cells. 1\#, Control siRNA cotransfected with wild-type PKD1. 2\#, PKD1 siRNA cotransfected with wild-type PKD1. 3\#, Control siRNA cotransfected with siRNA-resistant PKD1. 4\#, PKD1 siRNA cotransfected with siRNA-resistant PKD1. $\boldsymbol{L}$, Neurons cotransfected with dsRed and control siRNA. $\boldsymbol{M}$, Neurons cotransfected with dsRed and PKD1 siRNA. N, Neurons cotransfected with PKD1 siRNA and wild-type PKD1 resistant to siRNA. 0, Neurons cotransfected with PKD1 siRNA and dominant-negative PKD1 resistant to siRNA. $\boldsymbol{P}$, The percentage of neurons with multiple axons in each group transfected with the indicated siRNA or DNA constructs. Scale bars: $\boldsymbol{A}-\mathbf{C}, 40 \mu \mathrm{m} ; \mathbf{G}-\mathbf{I}, 20 \mu \mathrm{m} ; \boldsymbol{L}-\mathbf{0}, 20 \mu \mathrm{m}$.

not recover and only a small number of the neurons established normal polarity (percentage of polarized neurons: DMSO: $80.53 \pm 3.93 \%, n=270$ neurons; $1 \mu \mathrm{M}$ Gö6976: $9.9 \pm 1.19 \%, n=$ 252 neurons; three experiments), whereas most had multiple axons (Fig. 1D1,D2), indicating the irreversible effects of Gö6976. These results showed that the activity of PKD in early stage of neuronal development was important for the establishment of neuronal polarity. This is consistent with the previous opinion that the early stage of neuronal development ( $48 \mathrm{~h}$ after neuronal plating) is crucial for the formation of axon-dendrite polarity (Dotti et al., 1988; Jiang et al., 2005). In addition to inhibiting PKD, Gö6976 also inhibits the activity of conventional PKCs (Martiny-Baron et al., 1993). However, in cultured hippocampal neurons, conventional PKCs are initially present at very low levels and increase continuously over 2-3 d of culture (Tejero-Díez et al., 1995), by which time, most neurons have established polarity (Dotti et al., 1988). No significant effect on neuronal polarization was observed when the neurons were exposed to BIM, a conventional PKC inhibitor (data not shown), which is consistent with the results of previous studies (Shi et al., 2003). Thus, the role of conventional PKCs in neuronal polarization might be excluded.

The essential role of PKD1 in neuronal polarization was further confirmed by expressing constructs of GFP-tagged
dominant-negative-PKD1, D727A, in which Asp727 was replaced with alanine (Iglesias et al., 1998). Dissociated hippocampal neurons were transfected with either the GFP-DN-PKD1 construct or a GFP control construct. The neurons were then cultured for $2.5 \mathrm{~d}$ before analysis of axonal-dendritic polarity, by measuring the relative length of axons versus dendrites and staining with the axon-specific marker, Tau- 1 . The ratio of the length of the longest and second longest neurites (referred as polarity index) in neurons transfected with GFP alone was 7.15 \pm 0.64 (Fig. 2A,D) ( $n=113$ neurons; four experiments), and most of these neurons had a single axon (Fig. $2 G$ ). Transfection of neurons with wild-type (wt) PKD1 had no effect on neuronal polarity (polarity index: $7.46 \pm 1.93 ; n=38$ neurons; four experiments) (Fig. $2 B, D$ ) with most of the neurons having one axon (Fig. $2 H$ ). On the contrary, the polarity index of neurons transfected with GFP-DN-PKD1 was $1.57 \pm 0.16$ (Fig. $2 C, D)(n=51$ neurons; four experiments), and most of these neurons had multiple axons (Fig. 2I, arrows). These results were confirmed by another dominant-negative PKD1, K612W (Johannes et al., 1998), in which Lys612 was replaced with tryptophan (supplemental Fig. $\mathrm{S} 3 A, B$, available at www.jneurosci.org as supplemental material).

Next, we used PKD1 siRNA to confirm the present results by using PKD inhibitor and dominant-negative PKD1. Be- 


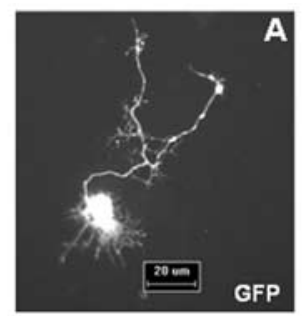

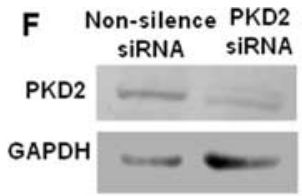
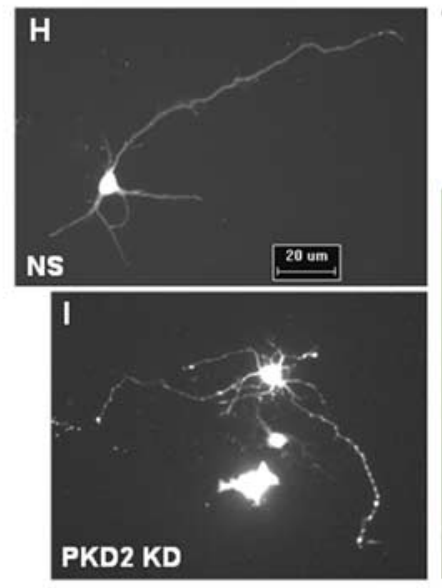

K

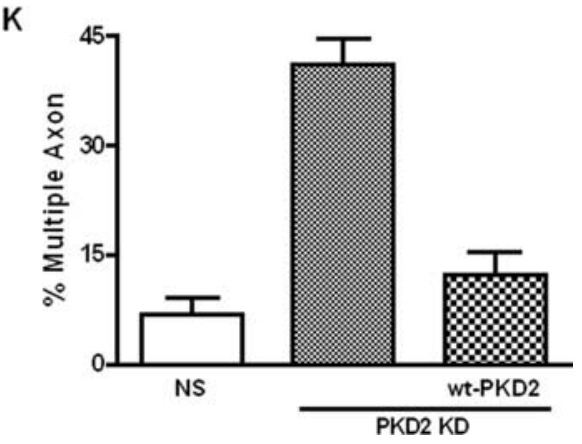

Figure 3. PKD2, but not PKD3, is involved in neuronal polarization. $\boldsymbol{A}-\boldsymbol{C}$, Neurons were transfected with GFP (A), GFP-DN-PKD2 (B1-B3), and GFP-DN-PKD3 (C1-C3), and were stained with anti-tubulin $\beta$ III. The arrows indicate axons. D, Percentage of polarized neurons transfected with GFP, GFP-DN-PKD2, and GFP-DN-PKD3. E, The polarity index in neurons transfected with GFP, GFP-DN-PKD2, and GFP-DN-PKD3. F, PKD2 siRNA knock down the expression of endogenous PKD2 in mouse neuroblastoma N2a cells. G, Western blot analyses of GFP-PKD2 in HEK293 cells. 1\#, Control siRNA cotransfected with wild-type PKD2. 2\#, PKD2 siRNA cotransfected with wild-type PKD2.3\#, Control siRNA cotransfected with siRNA-resistant PKD2. 4\#, PKD2 siRNA cotransfected with siRNA-resistant PKD2. $\boldsymbol{H}$, Neurons cotransfected with dsRed and control siRNA. I, Neurons cotransfected with dsRed and PKD2 siRNA.J, Neurons cotransfected with PKD2 siRNA and siRNA-resistant PKD2. $\boldsymbol{K}$, Percentage of neurons with multiple axons transfected with the indicated siRNA or DNA constructs. Bars indicate means \pm SEM of three independent experiments. Scale bars, $20 \mu \mathrm{m}$.

cause the gene sequence of PKD1 in rats was still unknown, we used mouse hippocampal neurons to conduct RNA interference experiments. PKD1 siRNA could efficiently knock down the expression of both the endogenous (Fig. $2 J$ ) and exogenous (Fig. $2 \mathrm{~K}$ ) mouse PKD1. dsRed plasmid was cotransfected with siRNA to identify the transfected cells. Transfection of control siRNA caused formation of very few neurons with multiple axons $(6.90 \pm 2.19 \% ; n=125$ neurons; three experiments) (Fig. $2 L, P$ ), whereas transfection of PKD1 siRNA markedly increased the percentage of neurons with multiple axons ( $41.73 \pm 1.2 \% ; n=131$ neurons; three experiments) (Fig. $2 M, P$ ). To avoid off-target effects of PKD1 siRNA on neuronal polarity, we did rescue experiments. Wild-type PKD1 resistant to PKD1 siRNA could rescue the formation of multiple axons caused by PKD1 siRNA (Fig. $2 N$ ), whereas dominant-negative PKD1 resistant to PKD1 siRNA (Fig. 2O) actually exaggerated the formation of multiple axons caused by PKD1 siRNA (percentage of neurons with multiple axons: PKD1 siRNA plus wt-PKD1: $9.52 \pm 1.19 \%, n=52$ neurons; PKD1 siRNA plus DN-PKD1: $63.07 \pm 1.96 \%, n=35$ neurons; three experiments) (Fig. $2 P$ ).
PKD2, but not PKD3, is required for neuronal polarization The PKD family consists of three isoforms: PKD1, PKD2, and PKD3 (Rozengurt et al., 2005). Recent studies have focused on isoform specificity in the control of membrane trafficking (Yeaman et al., 2004; Hausser et al., 2005; Sánchez-Ruiloba et al., 2006). Thus, we investigated whether PKD2 and PKD3 were also involved in neuronal polarization. Of neurons transfected with GFP alone, $89 \pm 1.73 \%$ had normal polarity (polarity index: $7.70 \pm 0.42 ; n=68$ neurons; three experiments) (Fig. $3 A, D, E$ ). In neurons transfected with GFP-tagged dominant-negative PKD2, only $21 \pm 4.16 \%$ of them had normal polarity (Fig. $3 D$ ), whereas most of them had multiple axons (Fig. 3B1,B2,B3, arrows) (polarity index: $1.87 \pm 0.19 ; n=58$ neurons; three experiments) (Fig. 3E). On the contrary, transfection with GFP-tagged dominant-negative PKD3 (Fig. 3C1,C2,C3) had no effect on neuronal polarization (percentage of polarized neurons: $78.33 \pm$ $11.61 \%$; polarity index: $4.27 \pm 0.18 ; n=34$ neurons; three experiments) (Fig. $3 D, E$ ). We also confirmed these results with RNA interference experiments. PKD2 siRNA could effectively knock down the expression of both endogenous (Fig. $3 F$ ) and exogenous (Fig. 3G) PKD2. Transfection with PKD2 siRNA 

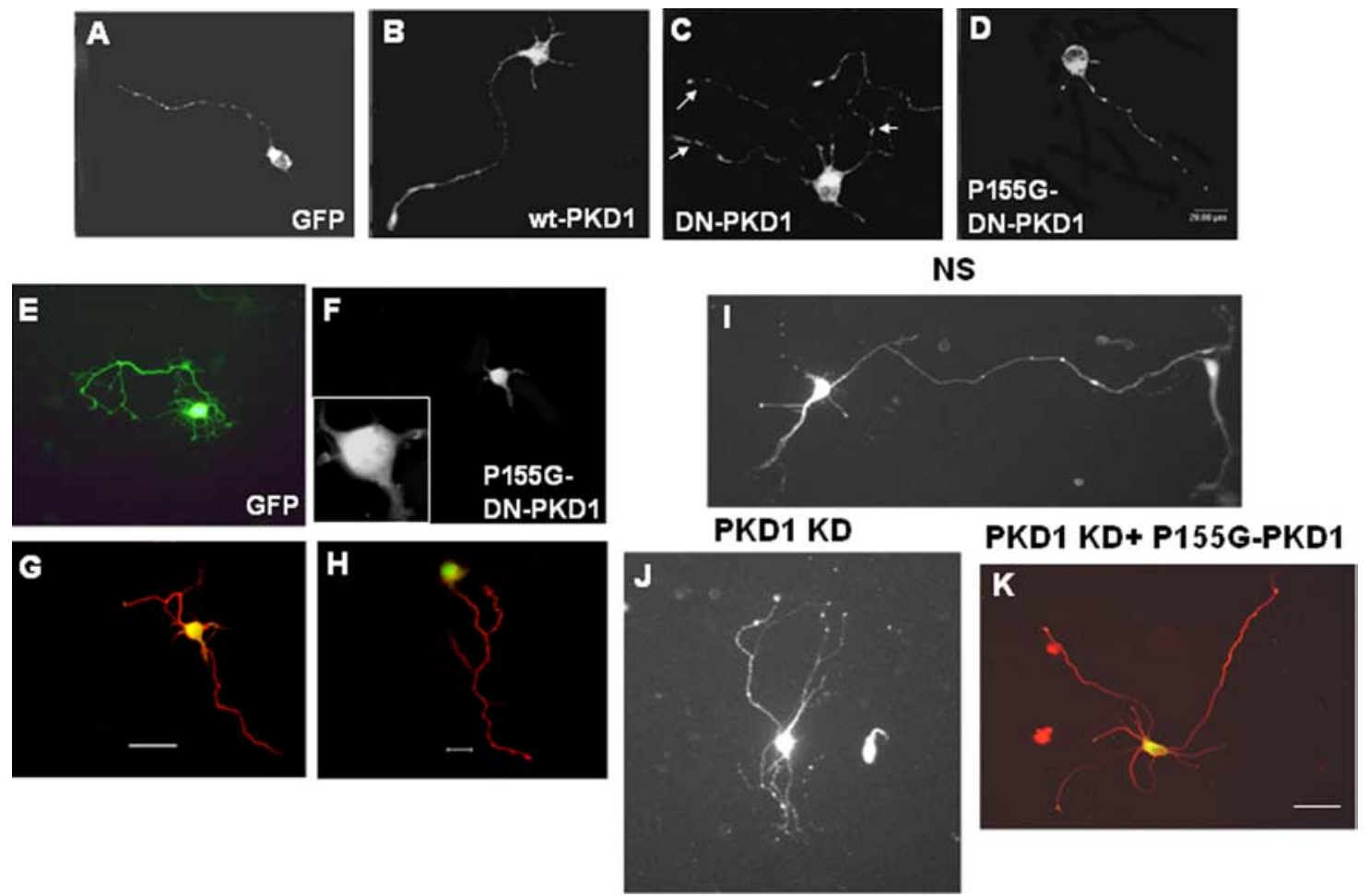

Figure 4. The effects of PKD1 on neuronal polarity depend on its activity in the Golgi apparatus. $\boldsymbol{A}-\boldsymbol{D}$, Post-Golgi membrane trafficking in neurons transfected with GFP (A), GFP-wt-PKD1 (B), GFP-DN-PKD1 ( $($ ) and GFP-P155G-DN-PKD1 (D). $\boldsymbol{E}$, Neurons transfected with GFP. $\boldsymbol{F}$, G, Neurons transfected with GFP-P155G-DN-PKD1 (F) were stained with anti-tubulin $\beta$ III $(\boldsymbol{G})$. $\boldsymbol{H}$, Neurons transfected with GFP-P155G-DN-PKD1 were stained with anti-Tau-1. I, Neurons cotransfected with dsRed and control siRNA. J, Neurons cotransfected with dsRed and PKD1 siRNA. $\boldsymbol{K}$, Neurons cotransfected with PKD1 siRNA and Golgi localization-deficient PKD1 resistant to PKD1 siRNA. Scale bars: $\boldsymbol{A}-\boldsymbol{D}, 20 \mu \mathrm{m} ; \boldsymbol{E}-\boldsymbol{G}, 40 \mu \mathrm{m} ; \boldsymbol{H}, 20 \mu \mathrm{m} ; \boldsymbol{I}-\boldsymbol{K}, 20 \mu \mathrm{m}$.

markedly increased the percentage of neurons with multiple axons compared with transfection with control siRNA (Fig. $3 H, I$ ) (control siRNA: $6.90 \pm 2.19 \%, n=125$ neurons; PKD2 siRNA: $41 \pm 3.69 \%, n=114$ neurons; three experiments) (Fig. $3 K$ ). To avoid off-target effects of siRNA, we also did rescue experiments and found that the effect of PKD2 siRNA on neuronal polarity could be rescued with siRNA-resistant GFP-PKD2 (Fig. 3J) (percentage of neurons with multiple axons: PKD2 siRNA plus siRNA-resistant GFP-PKD2: $12 \pm 3.15 \% ; n=62$ neurons; three experiments) (Fig. $3 K$ ).

\section{The effect of PKD1 on neuronal polarization depends on its} activity in the Golgi apparatus

As shown in Figure 2, E and F, GFP-DN-PKD1 increased the total number but not the total length of neurites per neuron, consistent with the results of Gö6976 treatment, suggesting that dominant-negative PKD1 specifically disrupts the polarization but not the total level of membrane trafficking in young hippocampal neurons. The following results support the above hypothesis that neurons transfected with GFP showed polarized post-Golgi membrane trafficking (Fig. $4 A$ ), dominant-negative PKD1 caused nonpolarized membrane trafficking in multiple directions (Fig. 4C, arrows), whereas neurons transfected with wild-type PKD1 (Fig. $4 B$ ) or dominant-negative PKD1 with defective Golgi localization (P155G-DN-PKD1) (Fig. 4D) maintained polarized membrane trafficking toward one neurite. P155G-DN-PKD1 showed no Golgi localization compared with DN-PKD1 (supplemental Fig. S3C,D, available at www.jneurosci.org as supplemental material).

Because PKD1 regulates membrane trafficking dependent on its activity in the Golgi apparatus, we asked whether the effects of PKD1 on neuronal polarity also depended on its activity in the
Golgi apparatus. First, in contrast to dominant-negative PKD1, which caused multiple axon formation, transfection with a dominant-negative PKD1 with defective Golgi localization (P155G-DN-PKD1) (Fig. 4F-H) had no effect on neuronal polarity (polarity index: $7.63 \pm 0.64 ; n=31$ neurons; three experiments). Moreover, P155G-DN-PKD1 did not increase the total number or the total length of neurites per neuron. Second, siRNA-resistant PKD1 with defective Golgi localization (P155GPKD1) could not rescue the formation of multiple axons caused by PKD1 siRNA (Fig. 4I-K) (percentage of neurons with multiple axons: PKD1 siRNA: $41.73 \pm 1.2 \%, n=131$ neurons; PKD1 siRNA plus P155G-PKD1: $39.93 \pm 0.46 \%, n=46$ neurons; three experiments; $p>0.05)$. Third, because PKD1 is localized to the Golgi apparatus via its C1a domain (Maeda et al., 2001) (Fig. 5A), we overexpressed a GFP-tagged C1a domain to compete with endogenous PKD1 for localization to the Golgi apparatus. Most neurons transfected with GFP alone had one axon (Fig. 5B), whereas neurons transfected with GFP-C1a had multiple axons (Fig. 5C). Statistical analyses indicated that transfection with GFP-C1a inhibited the formation of neuronal polarity (percentage of polarized neurons: GFP: $89 \pm 1.73 \%, n=68$ neurons; GFP-Cla: $8.67 \pm 3.93 \%, n=32$ neurons; three experiments; $p<$ 0.001; polarity index: GFP: $7.70 \pm 0.42$; GFP-C1a: $1.27 \pm 0.03$; three experiments; $p<0.001)$. All the above results indicated that the activity of PKD1 in the Golgi apparatus was important for neuronal polarization.

Overexpression of PKD1 can rescue the disruption of polarized membrane trafficking caused by cytochalasin $\mathrm{D}$ The above data showed that the lack of PKD1 function in the Golgi apparatus converted polarized into nonpolarized membrane trafficking in young hippocampal neurons. Next, we inves- 


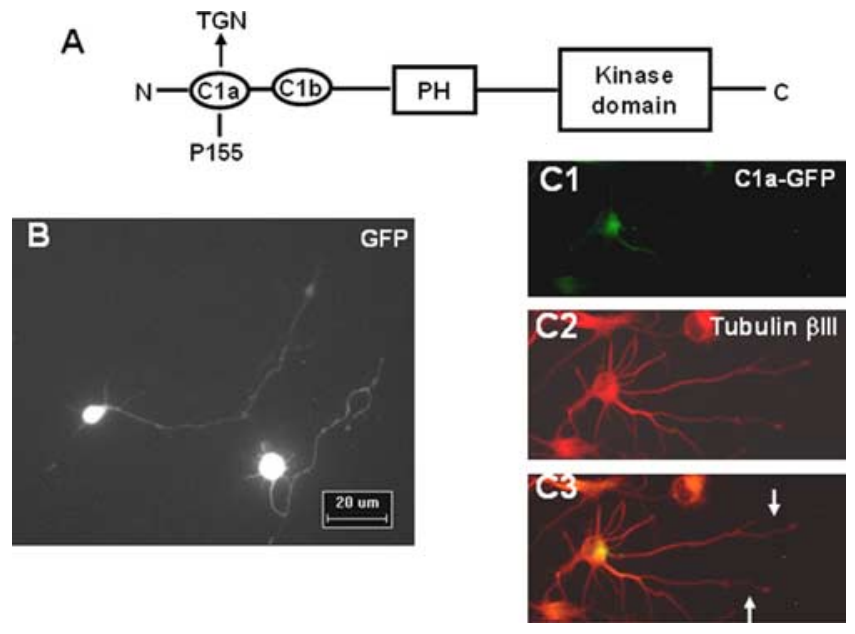

Figure 5. The effects of GFP-C1a on neuronal polarization. $A$, Schematic representation of the structure of PKD1. C1a and C1b, Domains of CRD (cysteine-rich domain); PH, pleckstrin homology domain. $\boldsymbol{B}, \boldsymbol{C}$, Neurons transfected with GFP $(\boldsymbol{B})$ and GFP-C1a $(\boldsymbol{C} \mathbf{C}-\mathbf{C})$ were stained with anti-tubulin $\beta$ III. The arrows indicate axons. Scale bar, $20 \mu \mathrm{m}$.

tigated the effects of PKD1 gain of function in the Golgi apparatus. Neurons were treated with $0.1 \mu \mathrm{M}$ cytochalasin D, an F-actin depolymerization agent, at $1 \mathrm{~d}$ in vitro for $24 \mathrm{~h}$. Most neurons lost F-actin in their growth cones compared with control neurons (supplemental Fig. S4A, B, arrows; available at www.jneurosci. org as supplemental material), consistent with previous findings (Bradke and Dotti, 1999). The disrupted neuronal polarity (Fig. $6 \mathrm{~A}$ ) and nonpolarized membrane trafficking (Fig. $6 \mathrm{~F}$ ) caused by cytochalasin $\mathrm{D}$ were both partially rescued by the overexpression of wild-type PKD1 (Fig. 6B,G). On the contrary, GFP-P155G$\mathrm{PKD} 1$, the construct with defective Golgi localization, failed to rescue the cytochalasin D phenotype (Fig. 6C), whereas GFPDN-PKD1 (Fig. 6D) actually exacerbated the disruption of neuronal polarity (percentage of polarized neurons: GFP plus cytochalasin D: $31.38 \pm 2.57 \%, n=104$ neurons; wt-PKD1 plus cytochalasin D: $64.97 \pm 1.73 \%, n=43$ neurons; P155G-PKD1 plus cytochalasin D: $31.13 \pm 3.21 \%, n=41$ neurons; DN-PKD1 plus cytochalasin D: $12.67 \pm 2.21 \%, n=39$ neurons; three experiments) (Fig. 6E). Interestingly, depolymerization of F-actin at the tips of neurites after cytochalasin D treatment was not rescued by overexpression of PKD1 (supplemental Fig. S4C,D, available at www.jneurosci.org as supplemental material). This was consistent with the data showing that PKD1 inhibition did not cause F-actin depolymerization at the tips of neurites (supplemental Fig. $\mathrm{S} 4 E, F$, available at www.jneurosci.org as supplemental material). These results suggested that overexpression of PKD1 could convert nonpolarized into polarized membrane trafficking through its activity in the Golgi apparatus and not via regulation of F-actin in neurites.

\section{PKD1 and PKD2 do not interact with the cytoskeleton}

All the above data indicated that PKD1 regulated neuronal polarity depending on its activity in the Golgi apparatus. Because most signaling proteins regulate neuronal polarity through their interactions with the cytoskeleton in neurites, we next investigated whether PKD1 interacted with the cytoskeleton. A detergent extraction experiment (Mascotti et al., 1997) showed that PKD1 immunolabeling was lost from the neuron after detergent extraction (supplemental Fig. S5A1,B1, available at www.jneurosci.org as supplemental material), indicating that PKD1 did not interact with the cytoskeleton. Western blot analysis also indicated that
PKD1 was only present in the Triton X-100-soluble fraction but not in the Triton X-100-insoluble fraction (supplemental Fig. $\mathrm{S} 5 C$, available at www.jneurosci.org as supplemental material). When a higher concentration of PKD1 antibody was used, PKD1 was still not detected (molecular weight, $115 \mathrm{kDa}$ ) in Triton $\mathrm{X}$-100-insoluble fraction, although nonspecific bands appeared (supplemental Fig. S5D, available at www.jneurosci.org as supplemental material). As a positive control, CDK5, which is known to interact with the cytoskeleton in neurons (Dhavan and Tsai, 2001), was detected in the Triton X-100-insolube fraction (supplemental Fig. S5E, available at www.jneurosci.org as supplemental material). Coimmunoprecipitation experiments showed that anti-PKD1 antibody could not immunoprecipitate actin or tubulin but could immunoprecipitate PKD1 and its substrate, N-cadherin (supplemental Fig. S5F, available at www. jneurosci.org as supplemental material). These results indicated that PKD1 did not interact with the cytoskeleton in young hippocampal neurons under basal conditions. We also could not detect the interaction of PKD2 with the cytoskeleton in Western blot analyses (supplemental Fig. S5G, available at www. jneurosci.org as supplemental material) or coimmunoprecipitation experiments (supplemental Fig. S5H, available at www. jneurosci.org as supplemental material).

Next, we examined whether PKD1 affected the stabilization of actin or microtubules in cell lines. Neither wild-type PKD1 nor dominant-negative PKD1 affected F-actin (supplemental Fig. $\mathrm{S} 6 A, B$, available at www.jneurosci.org as supplemental material) or microtubules (supplemental Fig. S6C,D, available at www. jneurosci.org as supplemental material) in Cos7 cells. Moreover, we did not detect the coimmunoprecipitation between PKD1 and actin or tubulin in Cos7 cells (data not shown). Finally, we addressed whether PKD1 influenced the polymerization of actin or microtubules in PC12 cells, which is a neuronal cell line. Neither wild-type PKD1 nor dominant-negative PKD1 affected F-actin (supplemental Fig. S7, available at www.jneurosci.org as supplemental material) or microtubules (data not shown) in PC12 cells.

\section{PKD1 and PKD2 are involved in the maintenance of neuronal polarity}

Neurons were cultured for $2.5 \mathrm{~d}$ until stage 3 before treatment with Gö6976, BIM, or DMSO for another $3 \mathrm{~d}$ and were fixed for immunostaining on day 5.5. The majority of neurons treated with DMSO (Fig. $7 A, E$ ) or BIM (Fig. $7 B$ ) on day 2.5 developed normal polarity by day 5.5. Gö6976 treatment from 60 to $132 \mathrm{~h}$ resulted in the formation of multiple axons (Fig. $7 C, D, F$ ). These results suggested that $\mathrm{PKD}$ inhibitor could affect the maintenance of neuronal polarity (percentage of polarized neurons: DMSO: $80.43 \pm 2.88 \%, n=225$ neurons; $1 \mu \mathrm{M}$ BIM: $77.20 \pm$ 7.42\%, $n=206$ neurons; $1 \mu \mathrm{M}$ Gö6976: $11.67 \pm 3.67 \%, n=215$ neurons; three experiments; polarity index: DMSO: $10.37 \pm 1.07$; $1 \mu \mathrm{M}$ Gö6976: $2.61 \pm 0.36)$. Gö6976 could also increase total neurite number and total neurite length with a minor but not statistically significant effect.

To further examine the role of PKD in the maintenance of neuronal polarity, we recorded individual neurites before and after Gö6976 treatment on day 2.5 and followed them through day 5.5. DMSO treatment did not influence the maintenance of neuronal polarity (Fig. $8 A, B$ ). As shown in Figure $8, C$ and $D$, there was one long neurite on day 2.5 (Fig. $8 C$, marked with a white arrow) and several shorter neurites on the same neuron. On day 5.5, Tau-1 and MAP2 staining showed that the originally long neurite (Fig. $8 D$, marked by a white arrow) was indeed an axon (Fig. $8 G$ ). Interestingly, two additional axons were formed 


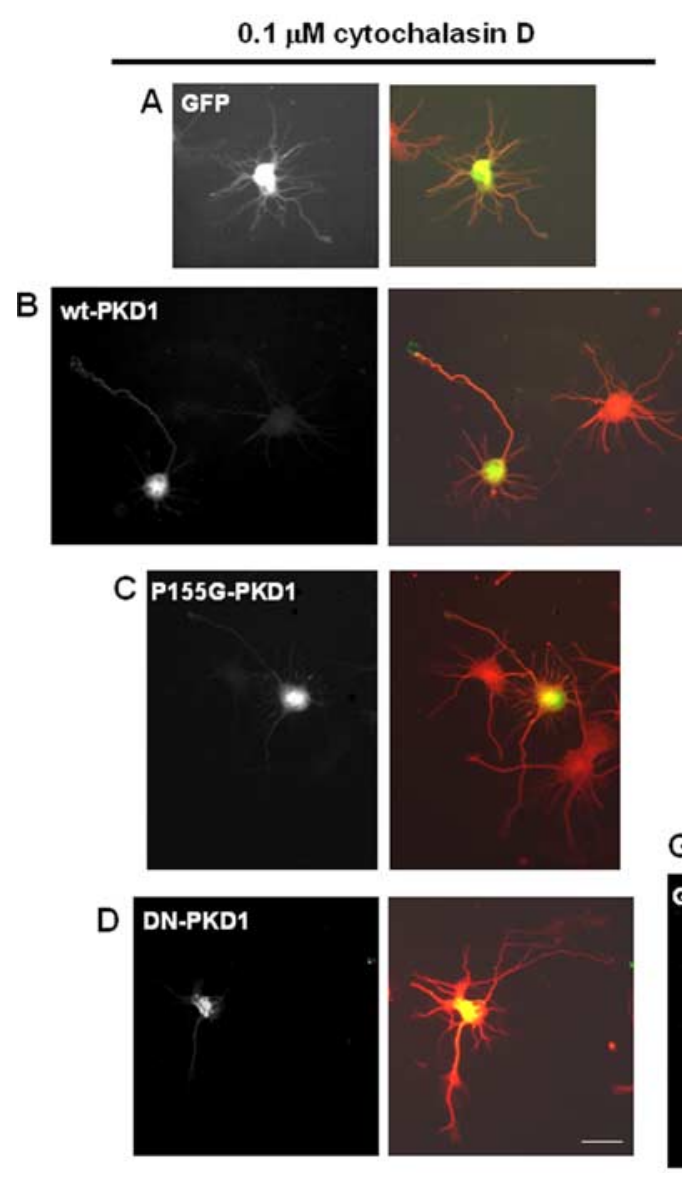

\section{$\mathrm{E}$}

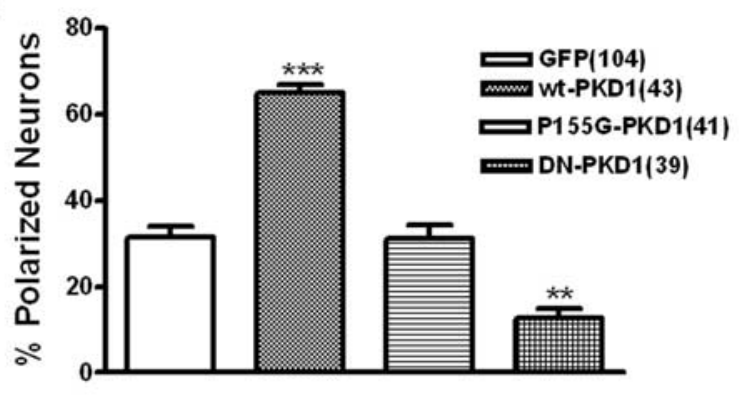

F $\quad$ GFP $+0.1 \mu \mathrm{M}$ cytochalasin D
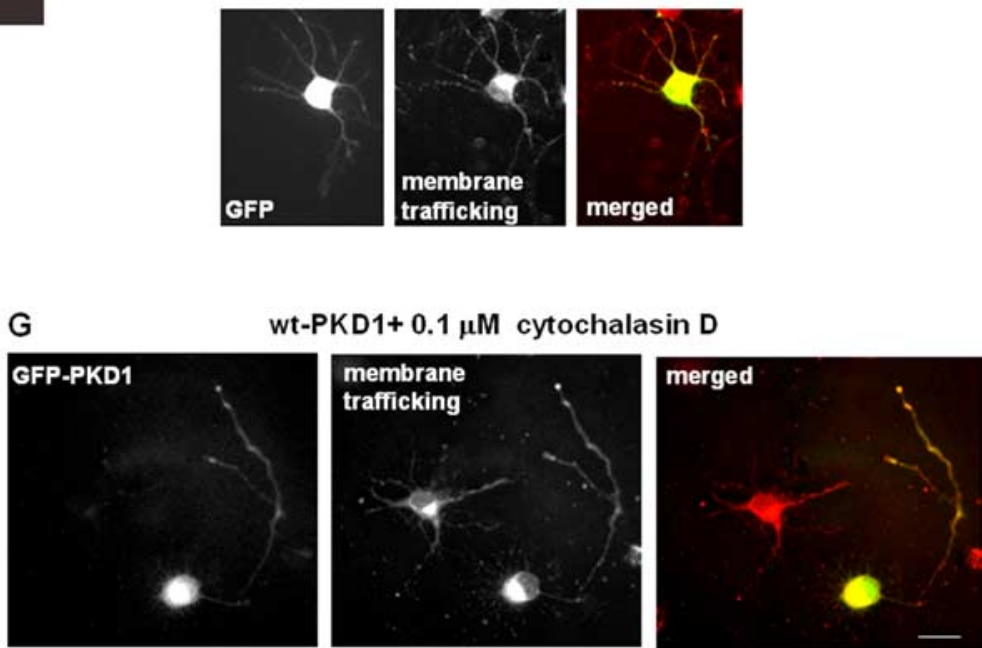

Figure 6. Overexpression of PKD1 could rescue the neuronal polarity and polarized membrane trafficking disrupted by cytochalasin D. $\boldsymbol{A}-\boldsymbol{D}$, Neurons expressing GFP (A), GFP-wt-PKD1 (B), GFP-P155G-PKD1 (C), and GFP-DN-PKD1 (D) were treated with $0.1 \mu \mathrm{m}$ cytochalasin D for $24 \mathrm{~h}$ and were stained with anti-tubulin $\beta$ III. $\boldsymbol{E}$, Percentage of polarized neurons expressing the indicated GFP fusion proteins under the role of cytochalasin $D$. The numbers in parentheses refer to the total neurons examined. Bars indicate means \pm SEM of three independent experiments. Data were analyzed by ANOVA followed by Newman-Keuls multiple-comparisons test. ${ }^{* *} p<0.01,{ }^{* * *} p<0.001$ compared with GFP group. $\boldsymbol{F}, \boldsymbol{G}$, Post-Golgi membrane trafficking in neurons expressing GFP (F) and GFP-wt-PKD1 (G). Scale bars: $A-D, 20 \mu \mathrm{m} ; \boldsymbol{F}, \boldsymbol{G}, 14 \mu \mathrm{m}$.

on day 5.5 (Fig. $8 D$, marked with red arrows; Fig. $8 G$, marked by white arrows) from two originally short neurites recorded on day 2.5 (Fig. 8C, marked with red arrows). These results directly demonstrated that inhibition of PKD activity could convert preexisting dendrites into axons (polarity index: DMSO group at day 2.5: $4.56 \pm 0.41 ;$ DMSO group at day 5.5: $9.8 \pm 2.99$; Gö6976 group at day 2.5: $4.77 \pm$ 0.92; Gö6976 group at day 5.5: $2.07 \pm$ 0.27; four experiments). The polarity index of neurons treated with DMSO increased by $113.7 \pm 64 \%$ from day 2.5 to day 5.5 , whereas the polarity index of neurons treated with $1 \mu \mathrm{M}$ Gö6976 decreased by $54.3 \pm 6 \%$ (four experiments).

The role of PKD in the maintenance of neuronal polarity was further confirmed by DNA transfection. Neurons were cotransfected with GFP fusion plasmids and dsRed on day 2 and cultured for another $3 \mathrm{~d}$, before fixing for immunostaining. Transfection with dominant-negative PKD1 (Fig. 9C) disrupted the maintenance of neuronal polarity and induced multiple axon formation, whereas transfection with wild-type PKD1 (Fig. 9B) or dominant-negative PKD1 with defective Golgi localization (Fig. 9D) did not affect the maintenance of neuronal polarity (percentage of neurons with multiple axons: dsRed: $11.67 \pm 1.67 \%, n=$ 110 neurons; dsRed plus wt-PKD1: $16.37 \pm 4.64 \%, n=78$ neurons; dsRed plus DN-PKD1: $68.03 \pm 4.07 \%, n=70$ neurons; dsRed plus P155G-DN-PKD1: $10.93 \pm 1.19 \%, n=68$ neurons; three experiments) (Fig. 9G). This result indicated that the main- tenance of neuronal polarity depended on the activity of PKD1 in the Golgi apparatus. Transfection with dominant-negative PKD2 (Fig. 9E), but not dominant-negative PKD3 (Fig. 9F), caused the formation of multiple axons (percentage of neurons with multiple axons: dsRed plus DN-PKD2: $68.57 \pm 3.31 \%, n=73$ neurons; dsRed plus DN-PKD3: $3.33 \pm 3.33 \%, n=31$ neurons; three experiments) (Fig. 9G). These data indicated that PKD2, but not PKD3, was involved in the maintenance of neuronal polarity. Next, we used siRNA to confirm the role of PKDs in the maintenance of neuronal polarity. Both PKD1 siRNA (Fig. 9I) and PKD2 siRNA (Fig. 9J) disrupted the maintenance of neuronal polarity by causing the formation of multiple axons (percentage of neurons with multiple axons: control siRNA: $3.47 \pm 1.61 \%$, $n=110$ neurons; PKD1 siRNA: $34.93 \pm 5.18 \%, n=115$ neurons; PKD2 siRNA: $32.5 \pm 5.2 \%, n=108$ neurons; three experiments) (Fig. 9K).

\section{Discussion}

Here, we show that PKD1 and PKD2 are essential for the establishment and maintenance of neuronal polarity. Loss of function of PKD in hippocampal neurons led to symmetric outgrowth of multiple axon-like processes. Underlying this symmetric neurite outgrowth was symmetric post-Golgi membrane trafficking. Because the net outgrowth of all neurites remained unchanged, we propose that PKD activity does not regulate the total level of 
membrane trafficking, but rather, its directionality. LIM kinase 1 (LIMK1) can also regulate the traffic of Golgi-derived vesicles (Rosso et al., 2004). However, PKD and LIMK1 likely have distinct roles in neuronal development because knockdown of LIMK1 also inhibits neurite growth, indicating that LIMK1 regulates overall level but not the polarity of membrane trafficking (Tursun et al., 2005). The present results are also different from the previous study indicating that disruption of the Golgi apparatus with brefeldin A (BFA) leads to no axon formation (Jareb and Banker, 1997). The difference might be reasonable because inhibition of PKD does not alter the integration of the Golgi apparatus (supplemental Fig. S3C, available at www.jneurosci.org as supplemental material), whereas BFA cause the Golgi apparatus to be dispersed.

To date, two protein kinases, GSK3 $\beta$ and MARK2 (microtubule affinityregulating kinase 2 ), have been reported whose lose of function can induce multiple axon formation (Jiang et al., 2005; Yoshimura et al., 2005; Chen et al., 2006) via their regulation of microtubule stability. Although a recent study indicated that the effects of GSK $3 \beta$ on neuronal polarity might be correlated with its Golgi localization (Gärtner et al., 2006), direct evidence is still lacking. Here, we provide the direct evidence that PKDs regulate neuronal polarization based on their activity in the Golgi apparatus. More interestingly, loss of function of PKD also influenced the maintenance of neuronal polarity. Underlying this effect was the conversion of preexisting dendrites into axons after PKD inhibition. We speculate that the axonspecific vesicles sorted by the Golgi apparatus might be mislocalized to dendrites on PKD inhibition, thus converting dendrites into axons. A recent study showed that deletion of a novel protein kinase, LRRK2 related kinase-1 (LRK-1), caused mislocalization of axon specific vesicles to dendrites in Caenorhabditis elegans (Sakaguchi-Nakashima et al., 2007). We speculated that LRK-1 regulated polarized vesicle trafficking through its activity in the Golgi apparatus. Interestingly, another study showed that the human homolog of LRK-1, leucine-rich repeat kinase-2 (LRRK2), regulated neurite process morphology in rat cortical neurons (MacLeod et al., 2006). Loss of function of LRRK2 in cortical neurons caused the formation of multiple axon-like processes, which was similar to the effects of PKD inhibition. These results support the hypothesis of how PKD regulates neuronal polarity in the Golgi apparatus. To further elucidate the mechanisms by which PKD regulates neubar, $20 \mu \mathrm{m}$.
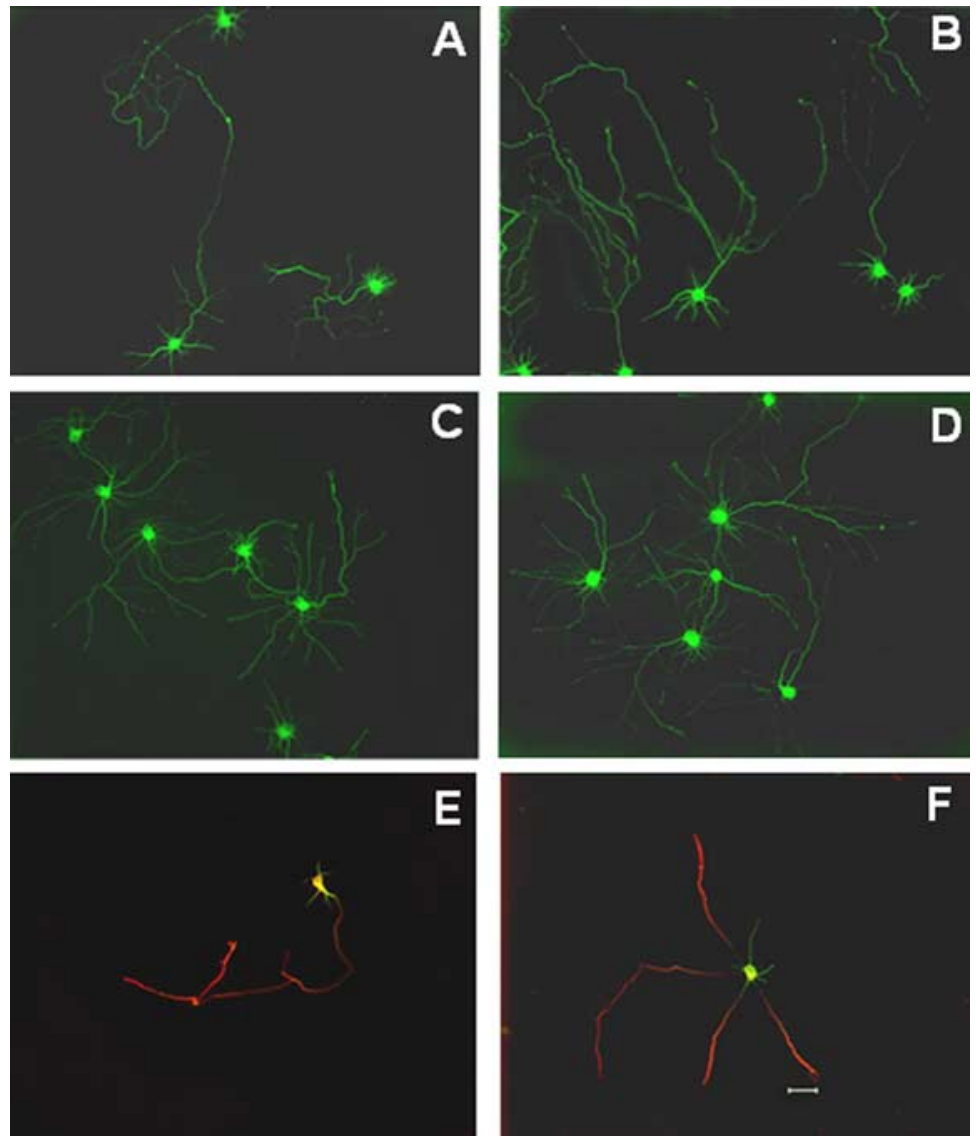

Figure 7. Effects of PKD inhibitor Gö6976 on the maintenance of neuronal polarity. $A-D$, Neurons treated with DMSO $(A), 0.3$ $\mu \mathrm{M}$ BIM (B), $0.3 \mu \mathrm{M}$ Gö6976 (C), and $1 \mu \mathrm{M}$ Gö6976 (D) were stained with anti-tubulin $\beta$ III. $\boldsymbol{E}$, $\boldsymbol{F}$, Neurons treated with DMSO (E) and $1 \mu \mathrm{M}$ Gö6976 (F) were stained with anti-MAP2 and anti-Tau-1. Scale bar, $20 \mu \mathrm{m}$.
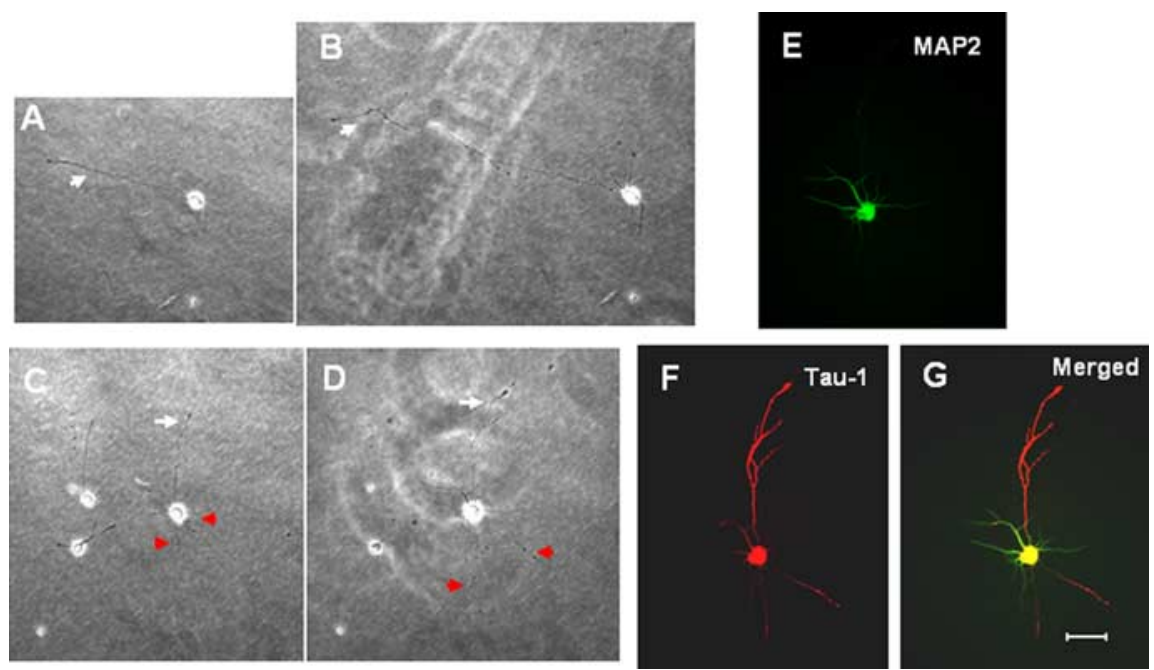

Figure 8. Conversion of preexisting dendrites into axons on PKD inhibition. $A, B$, The neuron before $(\boldsymbol{A})$ and after $(\boldsymbol{B})$ treatment with DMSO. The white arrows indicate axons. $\boldsymbol{C}, \boldsymbol{D}$, The neuron before $(\boldsymbol{C})$ and after $(\boldsymbol{D})$ treatment with $1 \mu \mathrm{m}$ Gö6976. The white arrow indicates the former axon, whereas the red arrows represent new axons produced from former dendrites. $E, F$, The neuron was stained with anti-MAP2 $(\boldsymbol{E})$ and anti-Tau-1 $(\boldsymbol{F}), \boldsymbol{G}, 0$ verlay of $\boldsymbol{E}$ and $\boldsymbol{F}$. The white arrows indicate the newly formed axons. Scale

ronal polarity, it is important to determine which Golgi-derived vesicles are involved in axon formation. Recent studies indicate that the selective translocation of the kinesin-1 motor marks the initial specification of the axon (Jacobson et al., 2006). Several 

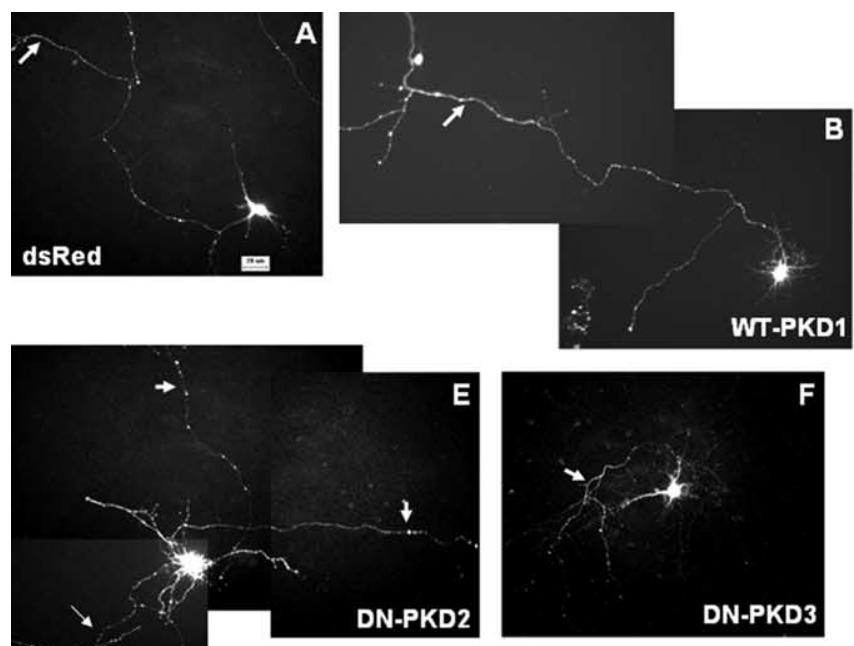

WT-PKD1
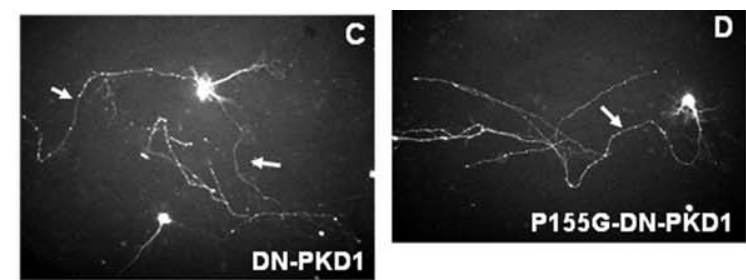

G
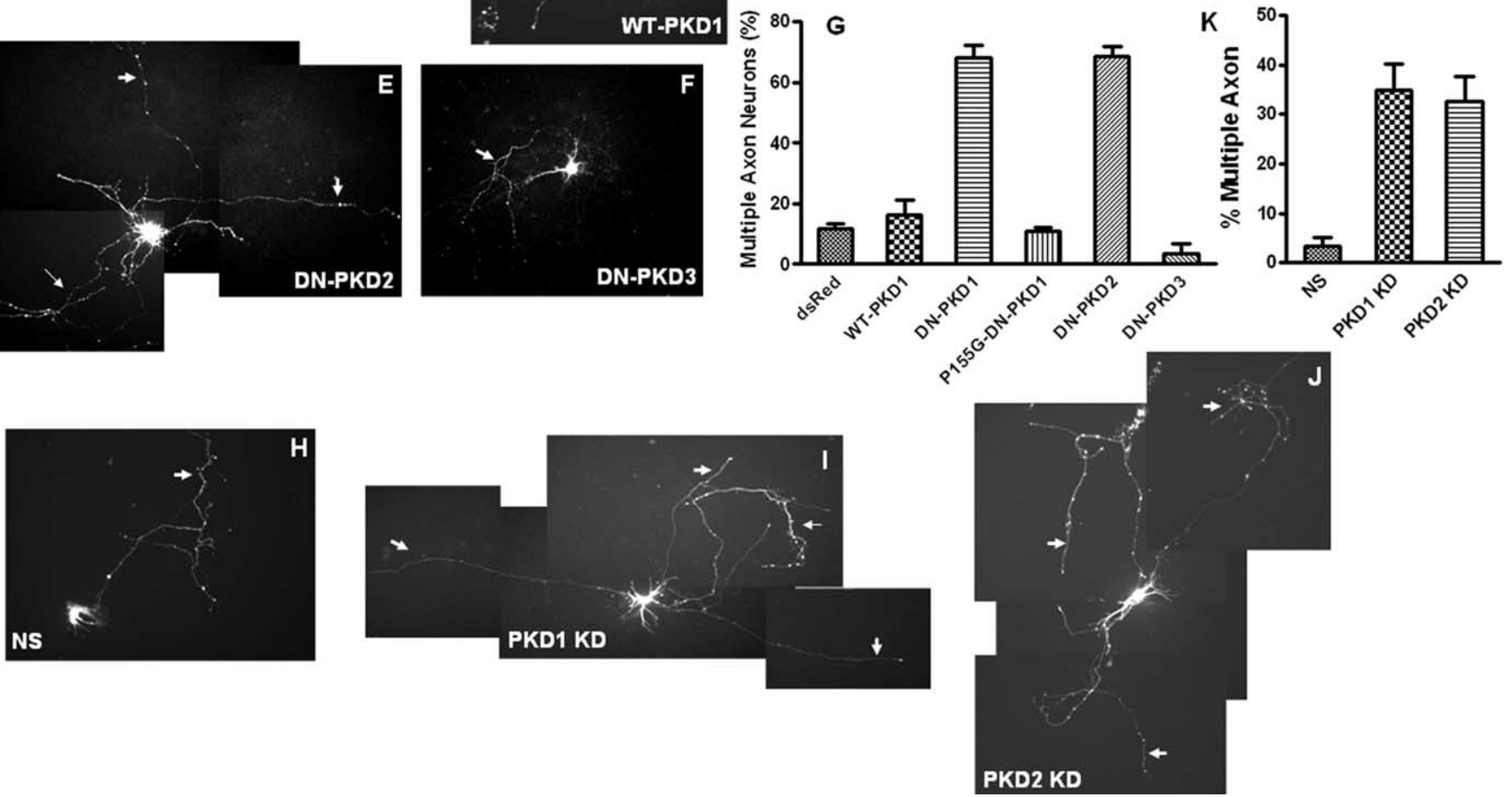

Figure 9. Effects of PKD mutants and PKD siRNA on the maintenance of neuronal polarity. $\boldsymbol{A}-\boldsymbol{F}$, Neurons cotransfected with dsRed (A) and GFP-wt-PKD1 (B), GFP-DN-PKD1 (C), GFP-P155G-DNPKD1 (D), GFP-DN-PKD2 (E), and GFP-DN-PKD3 (F). G, Percentage of neurons with multiple axons in each group transfected with the indicated constructs. Bars indicate means \pm SEM of three independent experiments. $\boldsymbol{H}-\boldsymbol{J}$, Mouse hippocampal neurons cotransfected with dsRed and control siRNA $(\boldsymbol{H})$, PKD1 siRNA $(\boldsymbol{I})$, and PKD2 siRNA $(\boldsymbol{J})$. $\boldsymbol{K}$, Percentage of neurons with multiple axons transfected with the indicated siRNA. Scale bar, $20 \mu \mathrm{m}$.

cargoes of kinesin-1, such as CRMP-2 (collapsin response mediator protein-2) (Kimura et al., 2005) and JIP-1 [JNK (c-Jun $\mathrm{N}$-terminal kinase)-interacting protein-1] (Muresan and Muresan, 2005) are found to be important in axon formation (Inagaki et al., 2001; Dajas-Bailador et al., 2008). However, the mechanisms underlying the selective accumulation of kinesin-1 motor in the axon are still unknown. It will be interesting to examine whether the polarized trafficking by kinesin- 1 is regulated by protein kinase D.

Although many studies focus on the role of PKD in regulating membrane trafficking in non-neural cells (Liljedahl et al., 2001; Prigozhina and Waterman-Storer, 2004; Woods et al., 2004; Yeaman et al., 2004; Sánchez-Ruiloba et al., 2006; Eiseler et al., 2007), very little is known about the functional significance of $P K D$ family in primary neurons. Here, we show that PKD1 and PKD2, but not PKD3 modulate the polarized mode but not the total level of membrane trafficking in stage 3 hippocampal neurons. Although the three DN-PKD mutants have similar consequences for TGN function in nonpolarized HeLa cells, our results together with the previous studies in polarized epithelial cells and neural cells demonstrate that PKD1 and PKD2 have different roles from PKD3 in regulating membrane trafficking. Several mechanisms might contribute to the phenomenon. First, PKD3 could regulate TGN-derived cargoes different from that of PKD1 and PKD2 in polarized epithelial cells (Yeaman et al., 2004). Sec- ond, the three PKDs might have different physiological substrates. For example, PKD1 and PKD2, but not PKD3 can phosphorylate phosphatidylinositol 4-phosphate kinase III $\beta$ (PI4KIII $\beta$ ) at Ser294 (Hausser et al., 2005). Because PI4KIII $\beta$ has also been implicated in Golgi-to-cell surface transport (Godi et al., 2004), this might be one of the mechanisms underlying the different roles of PKDs in regulating membrane trafficking. A recent study showed that PKD1 and PKD2 could phosphorylate a lipid transfer protein ceramide transport (CERT) in the Golgi (Fugmann et al., 2007). Phosphorylation on serine 132 by PKD decreases the affinity of CERT toward its lipid target phosphatidylinositol 4-phosphate (PI4P) at Golgi membranes and reduces ceramide transfer activity, identifying PKD as a regulator of lipid homeostasis. Whether PI4KIII $\beta$ and CERT are involved in neuronal polarization needs to be further addressed. Third, the postsynaptic density-95/Discs large/zona occludens-1 (PDZ)binding motif was identified in the $\mathrm{C}$ terminus of PKD1 and PKD2 but not in PKD3, which is responsible for the isoformspecific regulation in the transport of Kidins220 (a neural membrane protein identified as the first substrate of PKD1) (SánchezRuiloba et al., 2006). Considering that PDZ proteins control the asymmetrical delivery of membrane proteins from the TGN to the different plasma domains (Rongo, 2001), the C terminus PDZ-binding domain might constitute another mechanism underlying the different effects of PKDs on polarized membrane 
trafficking. Although PKD3 is not involved in polarity, it might regulate the growth of neurites. The primary data indicated that dominant-negative PKD3 could inhibit the neurites growth (neurites length of DIV2 neurons: GFP: $180.6 \pm 6.1 \mu \mathrm{m}, 45$ neurons; GFP-DN-PKD3: $103.1 \pm 18.1 \mu \mathrm{m}, 34$ neurons; three experiments; $p<0.05)$. A recent study indicated that PKD3 interacted with vesicle-associated membrane protein 2 (VAMP2) and regulated the localization of VAMP2 vesicles to plasma membrane (Lu et al., 2007). VAMP2 regulated fusion of vesicles with the plasma membrane (Kimura et al., 2003) and was involved in the neurites growth of PC12 cells (Shirasu et al., 2000).

It is intriguing that both $\mathrm{PKD} 1$ and $\mathrm{PKD} 2$ activity are required for neuronal polarity. On one hand, DN-PKD1 might affect the function of PKD2 in the Golgi by binding irreversibly to the TGN through DAG (Yeaman et al., 2004). Because PKD autophosphorylation is required for its release from the membrane (Maeda et al., 2001), this binding competitively inhibits the recruitment of endogenous PKD2 to the TGN. We think this interpretation is reasonable because the Golgi localization domains of the two PKDs are very similar. At the level of the amino acids, the C1a domain of PKD1 shows $92 \%$ identity to that of PKD2. So overexpression of PKD1-C1a might alter the Golgi localization of both PKD1 and PKD2. Our results support this hypothesis because PKD1-C1a has more dramatic effects on neuronal polarization than DN-PKD1 and DN-PKD2. However, both the PKD1 siRNA and PKD2 siRNA affect neuronal polarity, which indicates a partially nonredundant function of PKD1 and PKD2. This is similar to a recent study showing that depletion of either PKD2 or PKD3 affects Golgi-to-cell surface transport in HeLa cells that express only PKD2 and PKD3 but not PKD1 (Bossard et al., 2007). We proposed that PKD2 and PKD3 dimerize at the TGN to activate downstream targets. In hippocampal neurons, PKD1 and PKD2 might also need to be dimerized to regulate polarized membrane trafficking. Otherwise, the two PKD isoforms might have different substrates or target different pools of the same substrate in the Golgi. It is proposed that PKD1 and PKD2 may target distinct pools of PI4KIII $\beta$ at the TGN (Hausser et al., 2005). In support of this reasoning is the recent proposal that PI4P is located in functionally distinct TGN domains (Godi et al., 2004). PKD1 and PKD2 could be recruited to these separate domains at the TGN to regulate polarized trafficking of distinct cargo proteins. Finally, it might also be possible that reducing total PKD activity below a certain threshold is detrimental to polarization.

The mechanisms underlying polarized membrane trafficking can vary depending on the developmental stage of the neurons (Horton and Ehlers, 2003). Young neurons develop initial polarity by mechanisms analogous to those used by migrating cells (Bradke and Dotti, 1998), whereas mature neurons use the same machinery as polarized epithelial cells (Dotti and Simons, 1990). Thus, it will be interesting to investigate how $\mathrm{PKD}$ regulates membrane trafficking in mature hippocampal neurons. In fact, more and more studies indicate that some signaling proteins involved in the dendritic morphogenesis and synapse formation at the later stage of neuronal development also participate in neuronal polarization, such as the polarity proteins Par3, Par6, and aPKC (Shi et al., 2003; Zhang and Macara, 2006, 2008) and the Rho GTPases (Govek et al., 2005). It is reasonable to examine whether the members of PKD family are involved in the other processes of neuronal development such as axon targeting and synapse formation.

\section{References}

Arimura N, Kaibuchi K (2005) Key regulators in neuronal polarity. Neuron 48:881-884.

Arimura N, Kaibuchi K (2007) Neuronal polarity: from extracellular signals to intracellular mechanisms. Nat Rev Neurosci 8:194-205.

Barnes AP, Lilley BN, Pan YA, Plummer LJ, Powell AW, Raines AN, Sanes JR, Polleux F (2007) LKB1 and SAD kinases define a pathway required for the polarization of cortical neurons. Cell 129:549-563.

Baron CL, Malhotra V (2002) Role of diacylglycerol in PKD recruitment to the TGN and protein transport to the plasma membrane. Science 295:325-328.

Bossard C, Bresson D, Polishchuk RS, Malhotra V (2007) Dimeric PKD regulates membrane fission to form transport carriers at the TGN. J Cell Biol 179:1123-1131.

Bradke F, Dotti CG (1997) Neuronal polarity: vectorial cytoplasmic flow precedes axon formation. Neuron 19:1175-1186.

Bradke F, Dotti CG (1998) Membrane traffic in polarized neurons. Biochim Biophys Acta 1404:245-258.

Bradke F, Dotti CG (1999) The role of local actin instability in axon formation. Science 283:1931-1934.

Chen YM, Wang QJ, Hu HS, Yu PC, Zhu J, Drewes G, Piwnica-Worms H, Luo ZG (2006) Microtubule affinity-regulating kinase 2 functions downstream of the PAR-3/PAR-6/atypical PKC complex in regulating hippocampal neuronal polarity. Proc Natl Acad Sci U S A 103:8534-8539.

Craig AM, Banker G (1994) Neuronal polarity. Annu Rev Neurosci 17:267-310.

Dajas-Bailador F, Jones EV, Whitmarsh AJ (2008) The JIP1 scaffold protein regulates axonal development in cortical neurons. Curr Biol 18:221-226.

de Anda FC, Pollarolo G, Da Silva JS, Camoletto PG, Feiguin F, Dotti CG (2005) Centrosome localization determines neuronal polarity. Nature 436:704-708.

Dhavan R, Tsai LH (2001) A decade of CDK5. Nat Rev Mol Cell Biol 2:749-759.

Dotti CG, Simons K (1990) Polarized sorting of viral glycoproteins to the axon and dendrites of hippocampal neurons in culture. Cell 62:63-72.

Dotti CG, Sullivan CA, Banker GA (1988) The establishment of polarity by hippocampal neurons in culture. J Neurosci 8:1454-1468.

Eiseler T, Schmid MA, Topbas F, Pfizenmaier K, Hausser A (2007) PKD is recruited to sites of actin remodelling at the leading edge and negatively regulates cell migration. FEBS Lett 581:4279-4287.

Fugmann T, Hausser A, Schöffler P, Schmid S, Pfizenmaier K, Olayioye MA (2007) Regulation of secretory transport by protein kinase D-mediated phosphorylation of the ceramide transfer protein. J Cell Biol 178:15-22.

Gärtner A, Huang X, Hall A (2006) Neuronal polarity is regulated by glycogen synthase kinase-3 (GSK-3beta) independently of Akt/PKB serine phosphorylation. J Cell Sci 119:3927-3934.

Godi A, Di Campli A, Konstantakopoulos A, Di Tullio G, Alessi DR, Kular GS, Daniele T, Marra P, Lucocq JM, De Matteis MA (2004) FAPPs control Golgi-to-cell surface membrane traffic by binding to ARF and PtdIns(4)P. Nat Cell Biol 6:393-404.

Govek EE, Newey SE, Van Aelst L (2005) The role of the Rho GTPases in neuronal development. Genes Dev 19:1-49.

Gschwendt M, Gschwendt M, Dieterich S, Rennecke J, Kittstein W, Mueller HJ, Johannes FJ (1996) Inhibition of protein kinase $\mathrm{C} \mu$ by various inhibitors. Differentiation from protein kinase C isoenzyme. FEBS Lett 392:77-80.

Hausser A, Link G, Bamberg L, Burzlaff A, Lutz S, Pfizenmaier K, Johannes FJ (2002) Structural requirements for localization and activation of protein kinase $\mathrm{C} \mu$ at the Golgi compartment. J Cell Biol 156:65-74.

Hausser A, Storz P, Märtens S, Link G, Toker A, Pfizenmaier K (2005) Protein kinase $\mathrm{D}$ regulates vesicular transport by phosphorylating and activating phosphatidylinositol-4 kinase IIIbeta at the Golgi complex. Nat Cell Biol 7:880-886.

Horton AC, Ehlers MD (2003) Neuronal polarity and trafficking. Neuron 40:277-295.

Iglesias T, Waldron RT, Rozengurt E (1998) Identification of in vivo phosphorylation sites required for protein kinase D activation. J Biol Chem 273:27662-27667.

Inagaki N, Chihara K, Arimura N, Ménager C, Kawano Y, Matsuo N, Nishimura T, Amano M, Kaibuchi K (2001) CRMP-2 induces axons in cultured hippocampal neurons. Nat Neurosci 4:781-782.

Jacobson C, Schnapp B, Banker GA (2006) A change in the selective trans- 
location of the Kinesin-1 motor domain marks the initial specification of the axon. Neuron 49:797-804.

Jareb M, Banker G (1997) Inhibition of axonal growth by Brefeldin A in hippocampal neurons in culture. J Neurosci 17:8955-8963.

Jiang H, Guo W, Liang X, Rao Y (2005) Both the establishment and maintenance of neuronal polarity require active mechanisms: critical roles of GSK-3 $\beta$ and its upstream regulators. Cell 120:123-135.

Johannes FJ, Horn J, Link G, Haas E, Siemienski K, Wajant H, Pfizenmaier K (1998) Protein kinase $\mathrm{C} \mu$ downregulation of tumor-necrosis-factorinduced apoptosis correlates with enhanced expression of nuclear-factor$\kappa \mathrm{B}$-dependent protective genes. Eur J Biochem 257:47-54.

Kimura K, Mizoguchi A, Ide C (2003) Regulation of growth cone extension by SNARE proteins. J Histochem Cytochem 51:429-433.

Kimura T, Watanabe H, Iwamatsu A, Kaibuchi K (2005) Tubulin and CRMP-2 complex is transported via Kinesin-1. J Neurochem 93:1371-1382.

Kishi M, Pan YA, Crump JG, Sanes JR (2005) Mammalian SAD kinases are required for neuronal polarization. Science 307:929-932.

Liljedahl M, Maeda Y, Colanzi A, Ayala I, Van Lint J, Malhotra V (2001) Protein kinase D regulates the fission of cell surface destined transport carriers from the trans-Golgi network. Cell 104:409-420.

Lu G, Chen J, Espinoza LA, Garfield S, Toshiyuki S, Akiko H, Huppler A, Wang QJ (2007) Protein kinase D 3 is localized in vesicular structures and interacts with vesicle-associated membrane protein 2. Cell Signal 19:867-879.

MacLeod D, Dowman J, Hammond R, Leete T, Inoue K, Abeliovich A (2006) The familial Parkinsonism gene LRRK2 regulates neurite process morphology. Neuron 52:587-593.

Maeda Y, Beznoussenko GV, Lint JV, Mironov AA, Malhotra V (2001) Recruitment of protein kinase D to the trans-Golgi network via the first cysteine-rich domain. EMBO J 20:5982-5990.

Martiny-Baron G, Kazanietz MG, Mischak H, Blumberg PM, Kochs G, Hug H, Marmé D, Schächtele C (1993) Selective inhibition of protein kinase C isozymes by the indalocarbazole Gö6976. J Biol Chem 268:9194-9197.

Mascotti F, Cáceres A, Pfenninger KH, Quiroga S (1997) Expression and distribution of IGF-1 receptors containing a $\beta$-subunit variant $(\beta \mathrm{gc})$ in developing neurons. J Neurosci 17:1447-1459.

Muresan Z, Muresan V (2005) Coordinated transport of phosphorylated amyloid-beta precursor protein and c-Jun $\mathrm{NH}_{2}$-terminal kinaseinteracting protein-1. J Cell Biol 171:615-625.

Prigozhina NL, Waterman-Storer CM (2004) Protein kinase D-mediated anterograde membrane trafficking is required for fibroblast motility. Curr Biol 14:88-98.

Rongo C (2001) Disparate cell types use a shared complex of PDZ proteins for polarized protein localization. Cytokine Growth Factor Rev 12:349-359.

Rosso S, Bollati F, Bisbal M, Peretti D, Sumi T, Nakamura T, Quiroga S, Ferreira A, Cáceres A (2004) LIMK1 regulates Golgi dynamics, traffic of Golgi-derived vesicles, and process extension in primary cultured neurons. Mol Biol Cell 15:3433-3449.

Rozengurt E, Rey O, Waldron RT (2005) Protein kinase D signaling. J Biol Chem 280:13205-13208.

Sakaguchi-Nakashima A, Meir JY, Jin Y, Matsumoto K, Hisamoto N (2007)
LRK-1, a C. elegans PARK8-related kinase, regulates axonal-dendritic polarity of SV proteins. Curr Biol 17:592-598.

Sánchez-Ruiloba L, Cabrera-Poch N, Rodríguez-Martínez M, LópezMenéndez C, Jean-Mairet RM, Higuero AM, Iglesias T (2006) Protein kinase $\mathrm{D}$ intracellular localization and activity control kinase D-interacting substrate of 220-kDa traffic through a postsynaptic density95/discs large/zonula occludens-1-binding motif. J Biol Chem 281:18888-18900.

Shelly M, Cancedda L, Heilshorn S, Sumbre G, Poo MM (2007) LKB1/ STRAD promotes axon initiation during neuronal polarization. Cell 129:565-577.

Shi SH, Jan LY, Jan YN (2003) Hippocampal neuronal polarity specified by spatially localized mPar3/mPar6 and PI 3-kinase activity. Cell 112:63-75.

Shirasu M, Kimura K, Kataoka M, Takahashi M, Okajima S, Kawaguchi S, Hirasawa Y, Ide C, Mizoguchi A (2000) VAMP-2 promotes neurite elongation and SNAP-25A increases neurite sprouting in PC12 cells. Neurosci Res 37:265-275.

Tang BL (2001) Protein trafficking mechanisms associated with neurite outgrowth and polarized sorting in neurons. J Neurochem 79:923-930.

Tejero-Díez P, Rodríguez-Sánchez P, Díez-Guerra FJ (1995) Expression of protein kinase $\mathrm{C}$ isozymes in hippocampal neurons in culture. FEBS Lett 363:293-298.

Tursun B, Schlüter A, Peters MA, Viehweger B, Ostendorff HP, Soosairajah J, Drung A, Bossenz M, Johnsen SA, Schweizer M, Bernard O, Bach I (2005) The ubiquitin ligase Rnf6 regulates local LIM kinase 1 levels in axonal growth cones. Genes Dev 19:2307-2319.

Wang Y, Kedei N, Wang M, Wang QJ, Huppler AR, Toth A, Tran R, Blumberg PM (2004) Interaction between protein kinase $C \mu$ and the vanilloid receptor type 1. J Biol Chem 279:53674-53682.

Wiggin GR, Fawcett JP, Pawson T (2005) Polarity proteins in axon specification and synaptogenesis. Dev Cell 8:803-816.

Witte H, Neukirchen D, Bradke F (2008) Microtubule stabilization specifies initial neuronal polarization. J Cell Biol 180:619-632.

Woods AJ, White DP, Caswell PT, Norman JC (2004) PKD1/PKCmu promotes alphavbeta 3 integrin recycling and delivery to nascent focal adhesions. EMBO J 23:2531-2543.

Yeaman C, Ayala MI, Wright JR, Bard F, Bossard C, Ang A, Maeda Y, Seufferlein T, Mellman I, Nelson WJ, Malhotra V (2004) Protein kinase D regulates basolateral membrane protein exit from trans-Golgi network. Nat Cell Biol 6:106-112.

Yoshimura T, Kawano Y, Arimura N, Kawabata S, Kikuchi A, Kaibuchi K (2005) GSK-3beta regulates phosphorylation of CRMP-2 and neuronal polarity. Cell 120:137-149.

Yu W, Baas PW (1994) Changes in microtubule number and length during axon differentiation. J Neurosci 14:2818-2829.

Zhang H, Macara IG (2006) The polarity protein Par-3 and Tiam1 cooperate in dendritic spine morphogenesis. Nat Cell Biol 8:227-237.

Zhang H, Macara IG (2008) The PAR-6 polarity protein regulates dendritic spine morphogenesis through p190 RhoGAP and the Rho GTPase. Dev Cell 14:216-226.

Zmuda JF, Rivas RJ (1998) The Golgi apparatus and the centrosome are localized to the sites of newly emerging axons in cerebellar granule neurons in vitro. Cell Motil Cytoskeleton 41:18-38. 